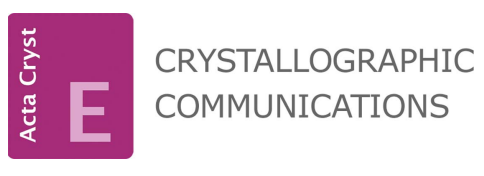

ISSN 2056-9890

Received 25 May 2016

Accepted 22 June 2016

Edited by M. Weil, Vienna University of Technology, Austria

Keywords: crystal structure; magnesiotaaffeite; gemstones; polytypism; polysomatism; modular structure.

CCDC reference: 1487140

Supporting information: this article has supporting information at journals.iucr.org/e

\section{Crystal structure of $\mathrm{Cr}$-bearing $\mathrm{Mg}_{3} \mathrm{BeAl}_{8} \mathrm{O}_{16}$, a new polytype of magnesiotaaffeite- $2 N^{\prime} 2 S$}

\author{
Thomas Malcherek $^{\mathrm{a} *}$ and Jochen Schlüter ${ }^{\mathrm{b}}$ \\ aMineralogisch-Petrographisches Institut, Universität Hamburg, Grindelallee 48, 20146 Hamburg, Germany, and

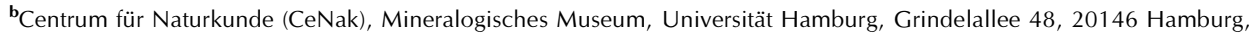 \\ Germany. *Correspondence e-mail: thomas.malcherek@uni-hamburg.de
}

The crystal structure of a new polytype of magnesiotaaffeite- $2 N^{\prime} 2 S$, ideally $\mathrm{Mg}_{3} \mathrm{BeAl}_{8} \mathrm{O}_{16}$ (trimagnesium beryllium octaaluminium hexadecaoxide), is described in space-group symmetry $P \overline{3} m 1$. It has been identified in a fragment of a mineral sample from Burma (Myanmar). The new polytype is composed of two $\mathrm{Mg}_{2} \mathrm{Al}_{4} \mathrm{O}_{8}(S)$ - and two $\mathrm{BeMgAl}_{4} \mathrm{O}_{8}\left(N^{\prime}\right)$-modules in a stacking sequence $N^{\prime} S S N^{\prime \prime}$ which differs from the $N^{\prime} S N^{\prime} S$-stacking sequence of the known magnesiotaaffeite- $2 N^{\prime} 2 S$ polytype. The crystal structure can be derived from a close-packed arrangement of $\mathrm{O}$ atoms and is discussed with regard to its polytypism and its $\mathrm{Cr}^{3+}$ chromophore content.

\section{Mineralogical and crystal-chemical context}

The minerals of the taaffeite group form a polysomatic series, composed of spinel $(S)$ and nolanite $\left(N^{\prime}\right)$ modules (Armbruster, 2002). The nolanite modules in the taaffeites are modified with respect to the nolanite, $(\mathrm{V}, \mathrm{Fe})_{5} \mathrm{O}_{7}(\mathrm{OH})$, crystal structure (Gatehouse et al., 1983), such that Be nominally substitutes for the hydrogen atoms of the nolanite $\mathrm{OH}$ group, while $\mathrm{Mg}$ and $\mathrm{Al}$ replace $\mathrm{V}$ and $\mathrm{Fe}$, respectively. Variable numbers of the $S$-modules, $\mathrm{Mg}_{2} \mathrm{Al}_{4} \mathrm{O}_{8}$, and of the $N^{\prime}$-modules, $\mathrm{BeMgAl}_{4} \mathrm{O}_{8}$, combine to yield different compositions of taaffeite minerals, i.e. different polysomes. Magnesiotaaffeite$2 N^{\prime} 2 S$ is composed of two modified nolanite modules $N^{\prime}$ and two spinel modules $S$, yielding an idealized composition of $\mathrm{Mg}_{3} \mathrm{BeAl}_{8} \mathrm{O}_{16}$. Be-doping of $\mathrm{MgAl}_{2} \mathrm{O}_{4}$ has been shown to cause growth of twinned spinel crystals as a precursor to the formation of magnesiotaaffeite polytypes (Drev et al., 2013).

Here we report the crystal structure of a new polytype of magnesiotaaffeite, magnesiotaaffeite- $2 N^{\prime} 2 S_{2}$ which differs from the known magnesiotaaffeite-2 $N^{\prime} 2 S$ (Nuber \& Schmetzer, 1983) by the module stacking sequence. The resulting space group symmetry is $P \overline{3} m 1$, as opposed to the $P 6_{3} m c$ symmetry of the previously known polytype.

\section{Structural commentary}

The crystal structure of the title compound is shown in Fig. 1. It can be described by the stacking of close-packed oxygen layers along [001], with layers of cations filling the interstices. Following the layer nomenclature of Nuber \& Schmetzer (1983), the ${ }^{[6]} \mathrm{Al},{ }^{[6]} \mathrm{Al} 3$ and ${ }^{[6]} \mathrm{Al} 4$ cations can be attributed to $O$-layers, the ${ }^{[6]} \mathrm{A} 15,{ }^{[4]} \mathrm{Mg} 1$ and ${ }^{[4]} \mathrm{Mg} 2$ cations to $T_{2}$-layers and 


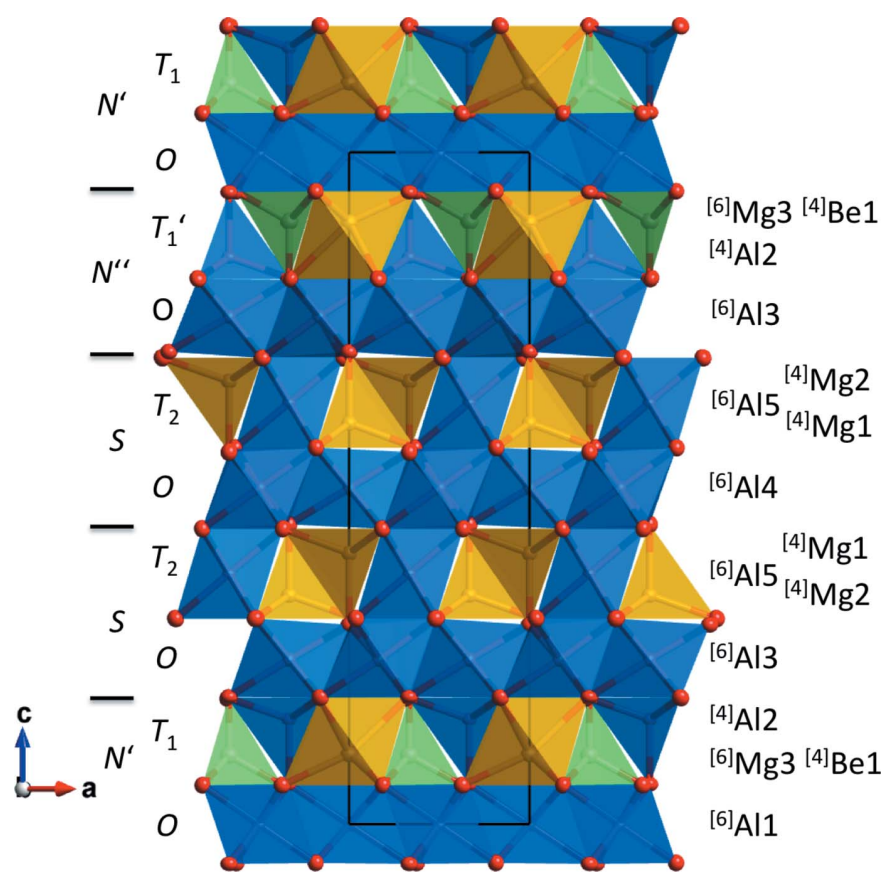

Figure 1

Polyhedral plot of magnesiotaaffeite- $2 N^{\prime} 2 S_{2}$ viewed down [010] with cation site nomenclature and coordination numbers given to the right. Module sequence is $N^{\prime}-S-S-N^{\prime \prime}-N^{\prime}$ from bottom to top, with boundaries indicated by horizontal lines. Displacement ellipsoids are drawn at the $99 \%$ probability level. $\mathrm{Mg}$ atoms are shown in yellow, $\mathrm{Al}$ in blue, $\mathrm{Be}$ in green and $\mathrm{O}$ in red.

the ${ }^{[4]} \mathrm{Be},{ }^{[4]} \mathrm{Al} 2$ and ${ }^{[6]} \mathrm{Mg} 3$ cations to $T_{1}$-layers. The cation stacking sequence is then $T_{1}-O-T_{2}-O-T_{2}-O-T_{1}{ }^{\prime} \cdots$ while the anion stacking sequence is $B A C B A C B C \cdots$ The orientation of $T_{1}{ }^{\prime}$ is upside down with respect to $T_{1}$. In the polytype described by Nuber \& Schmetzer (1983), the stacking sequence is $T_{1}-O-T_{2}-O-T_{1}-O-T_{2}-O \cdots$ and $B C A B C B A C \cdots$ by comparison. In terms of polysomatism, the $N^{\prime}$ layer is composed of one $T_{1}$ and one $O$-layer. The second nolanite layer, $N^{\prime \prime}$, is also composed of these layer types, but its $T_{1}$ layer is inverted with respect to the stacking direction. The $S$-layer is composed of one $O$-layer and one $T_{2}$-layer. Stacking these modules in the order $N^{\prime}-S-S-N^{\prime \prime}-N^{\prime}-\cdots$ generates the new polytype structure (Fig. 1). The stacking sequence of the known magnesiotaaffeite- $2 N^{\prime} 2 S$ polytype is $N^{\prime}-S-N^{\prime}-S-\cdots$.

The composition obtained by structure refinement is in good agreement with the composition obtained by electron microprobe analysis (EMPA). The calculated bond-valence sums agree reasonably well with the formal charges (Table 1), and on average they support the assumption that $\mathrm{Cr}$ is trivalent. Significant amounts of $\mathrm{Cr}^{3+}$ are found at the octahedrally coordinated $\mathrm{Al} 3$ and Al4-sites, where $\mathrm{Cr}^{3+}$ is overbonded, as well as at the tetrahedrally coordinated $\mathrm{Mg} 1$ and $\mathrm{Mg} 2$ sites, where $\mathrm{Cr}^{3+}$ is underbonded. $\mathrm{Cr}^{3+}$ in tetrahedral coordination is unusual, but has recently been reported for the brownmillerite-type compound $\mathrm{Ca}_{2} \mathrm{Cr}_{2} \mathrm{O}_{5}$ (Arevalo-Lopez \& Attfield, 2015) and for Cr-doped $\mathrm{BaAl}_{2} \mathrm{O}_{4}$ (Vrankić et al., 2015). However, without further confirmation by other methods, the appearance of tetrahedrally coordinated $\mathrm{Cr}^{3+}$ in the title
Table 1

Bond-valence sums (BVS).

Calculated using JANA2006 (Petřǐček et al., 2014) with bond-valence parameters taken from Brese \& O'Keeffe (1991). Angular brackets indicate site-occupancy weighted averages for the corresponding Mab sites.

\begin{tabular}{ll}
\hline Site & BVS \\
\hline Be1 & $1.956(5)$ \\
Al1 & $3.007(2)$ \\
Al2 & $2.788(3)$ \\
$\mathrm{Al} a$ & $2.932(2)$ \\
$\mathrm{Cr} 3 b$ & $3.571(3)$ \\
$<M 3 a b>$ & 2.943 \\
$\mathrm{Al} a$ & $2.955(2)$ \\
$\mathrm{Cr} 4 b$ & $3.599(3)$ \\
$<M 4 a b>$ & 2.966 \\
$\mathrm{Al} 5$ & $2.991(2)$ \\
$\mathrm{Mg} 1 a$ & $2.077(2)$ \\
$\mathrm{Cr} 1 b$ & $2.259(2)$ \\
$<M 1 a b>$ & 2.082 \\
$\mathrm{Mg} 2 a$ & $2.099(3)$ \\
$\mathrm{Cr} 2 b$ & $2.283(3)$ \\
$<M 2 a b>$ & 2.108 \\
$\mathrm{Mg} 3$ & $1.974(2)$ \\
$\mathrm{O} 1$ & $2.006(2)$ \\
$\mathrm{O} 2$ & $1.991(3)$ \\
$\mathrm{O} 3$ & $1.962(2)$ \\
O4 & $2.009(2)$ \\
$\mathrm{O} 5$ & $2.008(2)$ \\
O6 & $2.045(2)$ \\
O7 & $1.993(4)$ \\
O8 & $1.906(2)$ \\
\hline
\end{tabular}

compound should be treated with caution. The tetrahedral Mg1 coordination, with one $\mathrm{Mg} 1-\mathrm{O} 6$ distance of 1.9537 (12) $\AA$ and three Mg1-O4 distances of 1.9296 (7) $\AA$ is more distorted than the $\mathrm{Mg} 2$ coordination environment, where the longer Mg2-O1 distance [1.9361 (13) ̊] hardly differs from the three 1.9300 (7) $\AA \mathrm{Mg} 2$-O5 distances. The average bond lengths at the tetrahedral sites, nominally occupied by $\operatorname{Mg}(\operatorname{Mg} 11.936 \AA, M g 21.932 \AA)$, and at the octahedral sites, nominally occupied by Al (Al1 $1.909 \AA$ A, Al3 $1.916 \AA$, Al4 $1.913 \AA$, Al5 $1.909 \AA$ ), are similar to the $T$-O $(1.936 \AA)$ and $M$-O (1.923 $\AA$ ) distances reported for a natural $\mathrm{Cr}$ and $\mathrm{V}$-bearing spinel from Burma with a small inversion parameter (Widmer et al., 2015). This indicates that the degree of $\mathrm{Mg}, \mathrm{Al}$ disorder is equally low in the title compound. The $\mathrm{Al} 2$ site is at the center of a nearly regular oxygen tetrahedron with an average $\mathrm{Al}-\mathrm{O}$ distance of $1.785 \AA . \mathrm{Al}^{3+}$ is slightly underbonded at this site (Table 1), which might indicate admixture of $\mathrm{Mg}$ atoms. The slightly overbonded $\mathrm{Mg} 2$ site might accommodate the resulting Al-excess. The $\mathrm{Be}^{2+}$ cation forms one short bond with $\mathrm{O} 7$ [1.602 (2) §] and three longer bonds $[1.6615(13) \AA]$ with the O3-anions, while the tetrahedral angles are either $97.89(9)^{\circ}(\mathrm{O} 3-\mathrm{Be} 1-\mathrm{O} 3)$ or $119.45(7)^{\circ}(\mathrm{O} 7-\mathrm{Be} 1-\mathrm{O} 3)$. The $\mathrm{Mg}$ atom in the ${\mathrm{Mg} 3 \mathrm{O}_{6^{-}}}^{-}$ octahedron exhibits a strong out-of-centre distortion, away from the Al3-cation, to which it has a distance of only 3.0580 (7) А.

Rotation of the refined crystal structure by $60^{\circ}$ about [001] brings the bottom $O$-layer (Fig. 1) into the same orientation as the third $O$-layer of the unrotated structure. Thus a correspondingly rotated twin domain of the polytype structure can 
form a strain free boundary after the first $S$-layer of the module sequence as shown in Fig. 1. At the twin boundary this results in a module sequence $N^{\prime}-S-N^{\prime}-S$, corresponding to the previously described polytype.

\section{Sample details and EMPA}

The studied natural sample of magnesiotaaffeite $(m=0.95 \mathrm{~g})$ originates from Chaung-gyi, Mogok, Pyin-Oo-Lwin district, Burma (Myanmar). It has a red colour and a layered appearance (Fig. 2). A small fragment of the original sample was examined using single crystal X-ray diffraction. The same crystal fragment was subsequently prepared for electron microprobe analysis (EMPA) using a Cameca SX100 electron microprobe, operating in wavelength-dispersion mode at $15 \mathrm{kV}$ and $20 \mathrm{nA}$. Standards were $\mathrm{MgO}, \mathrm{Al}_{2} \mathrm{O}_{3}$ and $\mathrm{Cr}_{2} \mathrm{O}_{3}$. Based on 16 anions and the $\mathrm{Be}$ concentration from single-crystal X-ray structure refinement $(1 \mathrm{Be})$, the empirical chemical formula was determined as $\mathrm{Al}_{7.86} \mathrm{Be}_{1.0} \mathrm{Cr}_{0.19} \mathrm{Mg}_{2.93} \mathrm{O}_{16}$. The corresponding oxide composition (in wt\%) is $\mathrm{MgO} 20.82, \mathrm{Cr}_{2} \mathrm{O}_{3} 2.50, \mathrm{Al}_{2} \mathrm{O}_{3} 70.72, \mathrm{BeO}$ 4.41 , yielding a total of $98.45 \%$.

\section{Refinement}

Crystal data, data collection and structure refinement details are summarized in Table 2. The results of the EMPA indicate that the magnesiotaaffeite crystal contains significant amounts of $\mathrm{Cr}$. In order to accurately refine small $\mathrm{Cr}$-site populations against the major constituent elements $\mathrm{Al}$ and $\mathrm{Mg}$, intensities at small scattering angles were systematically weighted down by a factor of $1-\exp \left[-5(\sin \theta / \lambda)^{2}\right]$ in order to emphasize core electron contributions to the X-ray scattering. For that purpose, $\mathrm{Cr}$ and $\mathrm{Mg}$ or $\mathrm{Al}$ were constrained to have the same coordinates and displacement parameters under consideration

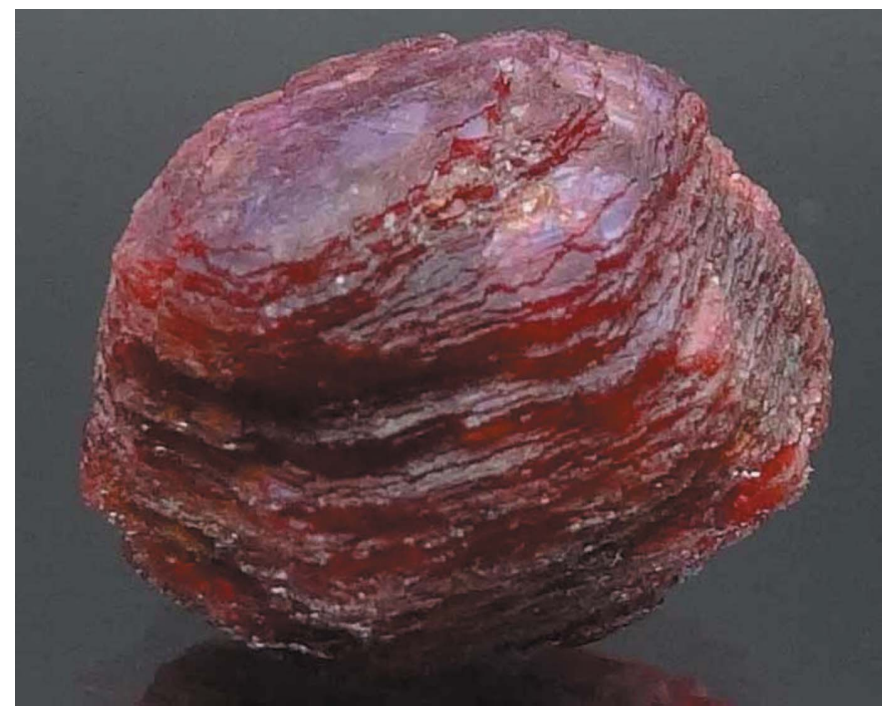

Figure 2

Magnesiotaaffeite sample, approximate size $1.0 \times 0.9 \times 0.8 \mathrm{~cm}$. (Photograph courtesy of Daniela Braith, Munich.)
Table 2

Experimental details.

\begin{tabular}{|c|c|}
\hline \multicolumn{2}{|l|}{ Crystal data } \\
\hline Chemical formula & $\mathrm{Mg}_{3} \mathrm{BeAl}_{8} \mathrm{O}_{16}$ \\
\hline$M_{\mathrm{r}}$ & 557.75 \\
\hline Crystal system, space group & Trigonal, $P \overline{3} m 1$ \\
\hline Temperature $(\mathrm{K})$ & 295 \\
\hline$a, c(\AA)$ & $5.6788(3), 18.3368(14)$ \\
\hline$V\left(\AA^{3}\right)$ & $512.11(7)$ \\
\hline$Z$ & 2 \\
\hline Radiation type & Мо $K \alpha$ \\
\hline$\mu\left(\mathrm{mm}^{-1}\right)$ & 1.25 \\
\hline Crystal size $(\mathrm{mm})$ & $0.23 \times 0.22 \times 0.10$ \\
\hline \multicolumn{2}{|l|}{ Data collection } \\
\hline Diffractometer & Nonius KappaCCD \\
\hline Absorption correction & $\begin{array}{l}\text { Multi-scan ( } S A D A B S \text {; Bruker, } \\
\text { 2008) }\end{array}$ \\
\hline$T_{\min }, T_{\max }$ & $0.614,0.747$ \\
\hline $\begin{array}{l}\text { No. of measured, independent and } \\
\text { observed }[I>2 \sigma(I)] \text { reflections }\end{array}$ & $10618,940,912$ \\
\hline$R_{\text {int }}$ & 0.040 \\
\hline$(\sin \theta / \lambda)_{\max }\left(\AA^{-1}\right)$ & 0.807 \\
\hline \multicolumn{2}{|l|}{ Refinement } \\
\hline$R\left[F^{2}>2 \sigma\left(F^{2}\right)\right], w R\left(F^{2}\right), S$ & $0.018,0.040,0.83$ \\
\hline No. of reflections & 940 \\
\hline No. of parameters & 75 \\
\hline$\Delta \rho_{\max }, \Delta \rho_{\min }\left(\mathrm{e} \AA^{-3}\right)$ & $0.41,-1.01$ \\
\hline
\end{tabular}

Computer programs: COLLECT (Nonius, 1998), EVAL15/Peakref and EVAL15 (Schreurs et al., 2010), SUPERFLIP (Palatinus \& Chapuis, 2007), SHELXL2014 (Sheldrick, 2015), VESTA (Momma \& Izumi, 2011) and publCIF (Westrip, 2010).

of full occupancy for the corresponding site. Scattering factors for neutral atoms were used and all atoms were refined with anisotropic displacement parameters. No evidence for mixed occupancy was found at the Be site; small $\mathrm{Cr}$ amounts were found for the $\mathrm{Al} 3, \mathrm{Al}, \mathrm{Mg} 1$ and $\mathrm{Mg} 2$ sites with occupation factors for $\mathrm{Cr}$ of 0.017 (3), 0.017 (5), 0.028 (5) and 0.048 (5), respectively. Two twin domains (twinning by merohedry) with volume fractions of 0.64 and 0.36 contribute to the total scattering intensity, related by reflection parallel to [1피 or, equivalently, by $60^{\circ}$ rotation about [001].

\section{Acknowledgements}

The sample material was kindly provided by D. Braith. The authors would like to thank S. Heidrich for providing the electron microprobe analysis and P. Stutz for sample preparation.

\section{References}

Arevalo-Lopez, A. \& Attfield, J. P. (2015). Dalton Trans. 44, 1066110664.

Armbruster, T. (2002). Eur. j. Miner. 14, 389-395.

Brese, N. E. \& O'Keeffe, M. (1991). Acta Cryst. B47, 192-197.

Bruker (2008). SADABS. Bruker AXS Inc., Madison, Wisconsin, USA.

Drev, S., Rečnik, A. \& Daneu, N. (2013). CrystEngComm, 15, 2640 2647.

Gatehouse, B. M., Grey, I. E. \& Nickel, E. H. (1983). Am. Mineral. 68, 833-839.

Momma, K. \& Izumi, F. (2011). J. Appl. Cryst. 44, 1272-1276.

Nonius (1998). COLLECT. Nonius BV, Delft, The Netherlands. 
Nuber, B. \& Schmetzer, K. (1983). Neues Jb. Miner. Monat. 393-402. Palatinus, L. \& Chapuis, G. (2007). J. Appl. Cryst. 40, 786-790.

Petř̌ček, V., Dušek, M. \& Palatinus, L. (2014). Z. Kristallogr. 229, 345-352.

Schreurs, A. M. M., Xian, X. \& Kroon-Batenburg, L. M. J. (2010). J. Appl. Cryst. 43, 70-82.
Sheldrick, G. M. (2015). Acta Cryst. C71, 3-8.

Vrankić, M., Gržeta, B., Lützenkirchen-Hecht, D., Bosnar, S. \& Šarić, A. (2015). Inorg. Chem. 54, 11127-11135.

Westrip, S. P. (2010). J. Appl. Cryst. 43, 920-925.

Widmer, R., Malsy, A.-K. \& Armbruster, T. (2015). Phys. Chem. Miner. 42, 251-260. 


\section{supporting information}

Acta Cryst. (2016). E72, 1060-1063 [https://doi.org/10.1107/S2056989016010215]

\section{Crystal structure of $\mathrm{Cr}$-bearing $\mathrm{Mg}_{3} \mathrm{BeAl}_{8} \mathrm{O}_{16}$, a new polytype of magnesiotaaffeite-2N'2S}

\section{Thomas Malcherek and Jochen Schlüter}

\section{Computing details}

Data collection: COLLECT (Nonius, 1998); cell refinement: EVAL15/Peakref (Schreurs et al., 2010); data reduction: EVAL15 (Schreurs et al., 2010); program(s) used to solve structure: SUPERFLIP (Palatinus \& Chapuis, 2007); program(s) used to refine structure: SHELXL2014 (Sheldrick, 2015); molecular graphics: VESTA (Momma \& Izumi, 2011); software used to prepare material for publication: publCIF (Westrip, 2010).

Trimagnesium beryllium octaaluminium hexadecaoxide

\section{Crystal data}

$\mathrm{Mg}_{3} \mathrm{BeAl}_{8} \mathrm{O}_{16}$

$M_{r}=557.75$

Trigonal, $P \overline{3} m 1$

$a=5.6788(3) \AA$

$c=18.3368(14) \AA$

$V=512.11(7) \AA^{3}$

$Z=2$

$F(000)=547$

\section{Data collection}

Nonius KappaCCD diffractometer

Radiation source: fine-focus sealed X-ray tube Graphite monochromator

Detector resolution: 9 pixels $\mathrm{mm}^{-1}$ $\varphi$ scans, and $\omega$ scans with $\kappa$ offsets

Absorption correction: multi-scan

(SADABS; Bruker, 2008)

$T_{\min }=0.614, T_{\max }=0.747$

\section{Refinement}

Refinement on $F^{2}$

Least-squares matrix: full

$R\left[F^{2}>2 \sigma\left(F^{2}\right)\right]=0.018$

$w R\left(F^{2}\right)=0.040$

$S=0.83$

940 reflections

75 parameters

0 restraints
$D_{\mathrm{x}}=3.617 \mathrm{Mg} \mathrm{m}^{-3}$

Mo $K \alpha$ radiation, $\lambda=0.71069 \AA$

Cell parameters from 9717 reflections

$\theta=2.2-35^{\circ}$

$\mu=1.25 \mathrm{~mm}^{-1}$

$T=295 \mathrm{~K}$

Tabular, red

$0.23 \times 0.22 \times 0.10 \mathrm{~mm}$

10618 measured reflections

940 independent reflections

912 reflections with $I>2 \sigma(I)$

$R_{\text {int }}=0.040$

$\theta_{\max }=35.0^{\circ}, \theta_{\min }=2.2^{\circ}$

$h=-8 \rightarrow 9$

$k=-9 \rightarrow 8$

$l=-29 \rightarrow 29$

$$
\begin{aligned}
& \left.w=\left\{1-\exp \left[-5(\sin \theta / \lambda)^{2}\right)\right]\right\} /\left[\sigma^{2}\left(F_{\mathrm{o}}^{2}\right)+(0.0296 P)^{2}\right. \\
& \quad+0.031 P] \\
& \quad \text { where } P=\left(F_{\mathrm{o}}^{2}+2 F_{\mathrm{c}}^{2}\right) / 3 \\
& (\Delta / \sigma)_{\max }<0.001 \\
& \Delta \rho_{\max }=0.41 \mathrm{e} \AA^{-3} \\
& \Delta \rho_{\min }=-1.01 \mathrm{e} \AA^{-3}
\end{aligned}
$$

Extinction correction: SHELXL2014

(Sheldrick, 2015),

$\mathrm{Fc}^{*}=\mathrm{kFc}\left[1+0.001 \mathrm{xFc}^{2} \lambda^{3} / \sin (2 \theta)\right]^{-1 / 4}$

Extinction coefficient: 0.046 (3) 


\section{Special details}

Geometry. All esds (except the esd in the dihedral angle between two 1.s. planes) are estimated using the full covariance matrix. The cell esds are taken into account individually in the estimation of esds in distances, angles and torsion angles; correlations between esds in cell parameters are only used when they are defined by crystal symmetry. An approximate (isotropic) treatment of cell esds is used for estimating esds involving l.s. planes.

Refinement. Refined as a 2-component twin

Fractional atomic coordinates and isotropic or equivalent isotropic displacement parameters $\left(\AA^{2}\right)$

\begin{tabular}{llllll}
\hline & $x$ & $y$ & $z$ & $U_{\mathrm{iso}} * / U_{\mathrm{eq}}$ & Occ. $(<1)$ \\
\hline Be1 & 0.3333 & 0.6667 & $0.10240(12)$ & $0.0054(3)$ & \\
$\mathrm{A} 11$ & 0.5000 & 0.0000 & 0.0000 & $0.00335(9)$ & \\
$\mathrm{A} 12$ & 0.6667 & 0.3333 & $0.15613(3)$ & $0.00344(9)$ & \\
A13A & $-0.33348(5)$ & $-0.16674(3)$ & $0.24468(2)$ & $0.00340(11)$ & $0.983(3)$ \\
Cr3B & $-0.33348(5)$ & $-0.16674(3)$ & $0.24468(2)$ & $0.00340(11)$ & $0.017(3)$ \\
A14A & 0.5000 & 0.0000 & 0.5000 & $0.00331(15)$ & $0.983(5)$ \\
Cr4B & 0.5000 & 0.0000 & 0.5000 & $0.00331(15)$ & $0.017(5)$ \\
A15 & 0.3333 & 0.6667 & $0.37276(3)$ & $0.00293(9)$ & \\
Mg1A & 0.0000 & 0.0000 & $0.40321(3)$ & $0.0040(2)$ & $0.972(5)$ \\
Cr1B & 0.0000 & 0.0000 & $0.40321(3)$ & $0.0040(2)$ & $0.028(5)$ \\
Mg2A & 0.6667 & 0.3333 & $0.34070(3)$ & $0.0041(2)$ & $0.952(5)$ \\
Cr2B & 0.6667 & 0.3333 & $0.34070(3)$ & $0.0041(2)$ & $0.048(5)$ \\
Mg3 & 0.0000 & 0.0000 & $0.10393(4)$ & $0.00473(11)$ & \\
O1 & 0.6667 & 0.3333 & $0.44628(7)$ & $0.00471(17)$ & \\
O2 & 0.6667 & 0.3333 & $0.05925(6)$ & $0.00435(18)$ & \\
O3 & $-0.81376(7)$ & $-0.18624(7)$ & $0.05785(4)$ & $0.00439(12)$ & \\
O4 & $-0.81598(7)$ & $-0.18402(7)$ & $0.43966(4)$ & $0.00449(12)$ & \\
O5 & $-0.51854(7)$ & $-0.03709(14)$ & $0.30594(3)$ & $0.00497(12)$ & \\
O6 & 0.0000 & 0.0000 & $0.29666(6)$ & $0.00458(18)$ & \\
O7 & 0.3333 & 0.6667 & $0.18977(6)$ & $0.00459(18)$ & \\
O8 & $-0.16175(6)$ & $-0.83825(6)$ & $0.18822(4)$ & $0.00461(11)$ & \\
& & & & & \\
\hline
\end{tabular}

Atomic displacement parameters $\left(\AA^{2}\right)$

\begin{tabular}{lllllll}
\hline & $U^{11}$ & $U^{22}$ & $U^{33}$ & $U^{12}$ & $U^{13}$ & $U^{23}$ \\
\hline Be1 & $0.0056(5)$ & $0.0056(5)$ & $0.0050(7)$ & $0.0028(2)$ & 0.000 & 0.000 \\
Al1 & $0.00339(12)$ & $0.00316(15)$ & $0.00343(15)$ & $0.00158(7)$ & $0.00049(6)$ & $0.00098(11)$ \\
A12 & $0.00375(12)$ & $0.00375(12)$ & $0.00281(18)$ & $0.00188(6)$ & 0.000 & 0.000 \\
Al3A & $0.00313(13)$ & $0.00356(12)$ & $0.00337(14)$ & $0.00157(7)$ & $0.00005(8)$ & $0.00003(4)$ \\
Cr3B & $0.00313(13)$ & $0.00356(12)$ & $0.00337(14)$ & $0.00157(7)$ & $0.00005(8)$ & $0.00003(4)$ \\
A14A & $0.00349(17)$ & $0.00302(19)$ & $0.00326(19)$ & $0.00151(10)$ & $0.00000(5)$ & $0.00000(11)$ \\
Cr4B & $0.00349(17)$ & $0.00302(19)$ & $0.00326(19)$ & $0.00151(10)$ & $0.00000(5)$ & $0.00000(11)$ \\
A15 & $0.00329(12)$ & $0.00329(12)$ & $0.00220(16)$ & $0.00165(6)$ & 0.000 & 0.000 \\
Mg1A & $0.0041(2)$ & $0.0041(2)$ & $0.0039(3)$ & $0.00204(12)$ & 0.000 & 0.000 \\
Cr1B & $0.0041(2)$ & $0.0041(2)$ & $0.0039(3)$ & $0.00204(12)$ & 0.000 & 0.000 \\
Mg2A & $0.0043(2)$ & $0.0043(2)$ & $0.0038(3)$ & $0.00215(11)$ & 0.000 & 0.000 \\
Cr2B & $0.0043(2)$ & $0.0043(2)$ & $0.0038(3)$ & $0.00215(11)$ & 0.000 & 0.000 \\
Mg3 & $0.00494(15)$ & $0.00494(15)$ & $0.0043(2)$ & $0.00247(8)$ & 0.000 & 0.000
\end{tabular}


supporting information

\begin{tabular}{lllllll} 
O1 & $0.0045(2)$ & $0.0045(2)$ & $0.0051(4)$ & $0.00226(12)$ & 0.000 & 0.000 \\
O2 & $0.0048(2)$ & $0.0048(2)$ & $0.0034(4)$ & $0.00241(12)$ & 0.000 & 0.000 \\
O3 & $0.00421(19)$ & $0.00421(19)$ & $0.0045(3)$ & $0.0019(2)$ & $0.00005(9)$ & $-0.00005(9)$ \\
O4 & $0.00431(19)$ & $0.00431(19)$ & $0.0049(3)$ & $0.0022(2)$ & $-0.00011(9)$ & $0.00011(9)$ \\
O5 & $0.00519(19)$ & $0.0047(2)$ & $0.0049(3)$ & $0.00234(12)$ & $0.00010(9)$ & $0.00020(18)$ \\
O6 & $0.0049(3)$ & $0.0049(3)$ & $0.0039(4)$ & $0.00247(13)$ & 0.000 & 0.000 \\
O7 & $0.0053(3)$ & $0.0053(3)$ & $0.0031(4)$ & $0.00267(13)$ & 0.000 & 0.000 \\
O8 & $0.00479(18)$ & $0.00479(18)$ & $0.0048(2)$ & $0.0028(2)$ & $-0.00033(10)$ & $0.00033(10)$ \\
\hline
\end{tabular}

Geometric parameters $\left(\AA,{ }^{\circ}\right)$

\begin{tabular}{|c|c|c|c|}
\hline $\mathrm{Be} 1-\mathrm{O} 7$ & $1.602(2)$ & $\operatorname{Mg} 1 \mathrm{~A}-\mathrm{A} 15^{\mathrm{xiii}}$ & $3.3259(2)$ \\
\hline $\mathrm{Be} 1-\mathrm{O} 3^{\mathrm{i}}$ & $1.6615(13)$ & $\mathrm{Mg} 1 \mathrm{~A}-\mathrm{Al} 3 \mathrm{~A}^{\mathrm{iv}}$ & $3.3376(6)$ \\
\hline $\mathrm{Be} 1-\mathrm{O}^{3 i}$ & $1.6615(13)$ & $\mathrm{Mg} 1 \mathrm{~A}-\mathrm{Cr} 3 \mathrm{~B}^{\mathrm{iv}}$ & $3.3376(6)$ \\
\hline $\mathrm{Be} 1-\mathrm{O} 3^{\mathrm{iii}}$ & $1.6615(13)$ & $\mathrm{Mg} 1 \mathrm{~A}-\mathrm{Al} 3 \mathrm{~A}^{\mathrm{iii}}$ & $3.3376(6)$ \\
\hline $\mathrm{Be} 1-\mathrm{Al} 1^{\mathrm{iv}}$ & $2.4926(17)$ & $\mathrm{Mg} 1 \mathrm{~A}-\mathrm{Cr} 3 \mathrm{~B}^{\mathrm{iii}}$ & $3.3376(6)$ \\
\hline $\mathrm{Be} 1-\mathrm{Al1}^{\mathrm{v}}$ & $2.4926(17)$ & $\mathrm{Mg} 2 \mathrm{~A}-\mathrm{O} 5^{\mathrm{viii}}$ & $1.9300(7)$ \\
\hline $\mathrm{Be} 1-\mathrm{A} 11^{\mathrm{vi}}$ & $2.4926(17)$ & $\mathrm{Mg} 2 \mathrm{~A}-\mathrm{O} 5^{\text {xiv }}$ & $1.9300(7)$ \\
\hline $\mathrm{Al} 1-\mathrm{O}^{\mathrm{vii}}$ & $1.8799(5)$ & $\mathrm{Mg} 2 \mathrm{~A}-\mathrm{O} 5^{\mathrm{iii}}$ & $1.9300(7)$ \\
\hline $\mathrm{Al1}-\mathrm{O} 3^{\mathrm{viii}}$ & $1.8799(5)$ & $\mathrm{Mg} 2 \mathrm{~A}-\mathrm{O} 1$ & $1.9361(13)$ \\
\hline $\mathrm{A} 11-\mathrm{O} 3^{\mathrm{ix}}$ & $1.8799(5)$ & $\mathrm{Mg} 2 \mathrm{~A}-\mathrm{A} 15^{\text {viii }}$ & $3.3310(2)$ \\
\hline $\mathrm{A} 11-\mathrm{O}^{\mathrm{x}}$ & $1.8799(5)$ & $\mathrm{Mg} 2 \mathrm{~A}-\mathrm{A} 15^{\mathrm{xiii}}$ & $3.3310(2)$ \\
\hline $\mathrm{A} 11-\mathrm{O} 2$ & $1.9666(6)$ & $\mathrm{Mg} 2 \mathrm{~A}-\mathrm{Al} 3 \mathrm{~A}^{\mathrm{iv}}$ & $3.3410(3)$ \\
\hline $\mathrm{A} 11-\mathrm{O} 2^{\mathrm{xi}}$ & $1.9667(6)$ & $\mathrm{Mg} 2 \mathrm{~A}-\mathrm{Al} 3 \mathrm{~A}^{\mathrm{viii}}$ & $3.3410(3)$ \\
\hline $\mathrm{A} 11-\mathrm{Be} 1^{\mathrm{xii}}$ & $2.4926(17)$ & $\mathrm{Mg} 2 \mathrm{~A}-\mathrm{A} 13 \mathrm{~A}^{\mathrm{xvi}}$ & $3.3410(3)$ \\
\hline $\mathrm{A} 11-\mathrm{Be} 1^{\mathrm{xiii}}$ & $2.4926(17)$ & $\mathrm{Mg} 2 \mathrm{~A}-\mathrm{A} 13 \mathrm{~A}^{\mathrm{xxiv}}$ & $3.3410(3)$ \\
\hline $\mathrm{All}-\mathrm{Al} 1^{\mathrm{xiv}}$ & $2.8394(2)$ & $\mathrm{Mg} 2 \mathrm{~A}-\mathrm{Al} 3 \mathrm{~A}^{\mathrm{iii}}$ & $3.3410(3)$ \\
\hline $\mathrm{A} 11-\mathrm{Al}^{\mathrm{xv}}$ & $2.8394(1)$ & $\mathrm{Mg} 3-\mathrm{O}^{\text {xvii }}$ & $2.0173(7)$ \\
\hline $\mathrm{Al1}-\mathrm{Al1}{ }^{\mathrm{vi}}$ & $2.8394(2)$ & $\mathrm{Mg} 3-\mathrm{O} 3^{\mathrm{ii}}$ & $2.0173(7)$ \\
\hline $\mathrm{A} 11-\mathrm{A} 11^{\mathrm{xvi}}$ & $2.8394(1)$ & $\mathrm{Mg} 3-\mathrm{O}^{\text {viii }}$ & $2.0173(7)$ \\
\hline $\mathrm{A} 12-\mathrm{O} 2$ & $1.7765(12)$ & $\mathrm{Mg} 3-\mathrm{O} 8^{\mathrm{xvi}}$ & $2.2181(8)$ \\
\hline $\mathrm{A} 12-\mathrm{O}^{\mathrm{i}}$ & $1.7874(7)$ & $\mathrm{Mg} 3-\mathrm{O}^{\mathrm{v}}$ & $2.2181(8)$ \\
\hline $\mathrm{Al} 2-\mathrm{O}^{\text {iv }}$ & $1.7874(7)$ & $\mathrm{Mg} 3-\mathrm{O} 8^{\mathrm{xviii}}$ & $2.2181(8)$ \\
\hline $\mathrm{Al} 2-\mathrm{O}^{\mathrm{xvi}}$ & $1.7874(7)$ & $\mathrm{Mg} 3-\mathrm{Al} 3 \mathrm{~A}^{\mathrm{iii}}$ & $3.0580(7)$ \\
\hline $\mathrm{A} 13 \mathrm{~A}-\mathrm{O} 6$ & $1.8969(6)$ & $\mathrm{Mg} 3-\mathrm{Cr} 3 \mathrm{~B}^{\mathrm{iii}}$ & $3.0580(7)$ \\
\hline $\mathrm{A} 13 \mathrm{~A}-\mathrm{O} 5^{\mathrm{xvii}}$ & $1.9189(5)$ & $\mathrm{Mg} 3-\mathrm{Al} 3 \mathrm{~A}^{\mathrm{iv}}$ & $3.0580(7)$ \\
\hline $\mathrm{A} 13 \mathrm{~A}-\mathrm{O} 5$ & $1.9189(5)$ & $\mathrm{Mg} 3-\mathrm{Cr} 3 \mathrm{~B}^{\mathrm{iv}}$ & $3.0580(7)$ \\
\hline $\mathrm{A} 13 \mathrm{~A}-\mathrm{O}^{\mathrm{v}}$ & $1.9193(5)$ & $\mathrm{O} 1-\mathrm{Cr}^{4} \mathrm{~B}^{\mathrm{xv}}$ & $1.9125(6)$ \\
\hline $\mathrm{A} 13 \mathrm{~A}-\mathrm{O} 8^{\mathrm{xviii}}$ & $1.9193(5)$ & $\mathrm{O} 1-\mathrm{Al}_{4} \mathrm{~A}^{\mathrm{xv}}$ & $1.9125(6)$ \\
\hline $\mathrm{A} 13 \mathrm{~A}-\mathrm{O} 7^{\mathrm{xix}}$ & $1.9232(6)$ & $\mathrm{O} 1-\mathrm{Cr} 4 \mathrm{~B}^{\mathrm{vi}}$ & $1.9125(6)$ \\
\hline $\mathrm{Al} 3 \mathrm{~A}-\mathrm{Cr} 3 \mathrm{~B}^{\mathrm{xvii}}$ & $2.8381(5)$ & $\mathrm{O} 1-\mathrm{Al}_{4} \mathrm{~A}^{\mathrm{vi}}$ & $1.9125(6)$ \\
\hline $\mathrm{A} 13 \mathrm{~A}-\mathrm{A} 13 \mathrm{~A}^{\mathrm{xvii}}$ & $2.8381(5)$ & $\mathrm{O} 2-\mathrm{A} 11^{\mathrm{xv}}$ & $1.9666(6)$ \\
\hline $\mathrm{A} 13 \mathrm{~A}-\mathrm{A} 13 \mathrm{~A}^{\mathrm{xx}}$ & $2.8381(5)$ & $\mathrm{O} 2-\mathrm{A} 11^{\mathrm{vi}}$ & $1.9666(6)$ \\
\hline $\mathrm{Al} 3 \mathrm{~A}-\mathrm{Cr} 3 \mathrm{~B}^{\mathrm{xx}}$ & $2.8381(5)$ & $\mathrm{O} 3-\mathrm{Be} 1^{\mathrm{xix}}$ & $1.6616(13)$ \\
\hline $\mathrm{Al} 3 \mathrm{~A}-\mathrm{Cr} 3 \mathrm{~B}^{\mathrm{iii}}$ & $2.8407(5)$ & $\mathrm{O} 3-\mathrm{A} 11^{\text {xviii }}$ & $1.8799(5)$ \\
\hline $\mathrm{Al} 3 \mathrm{~A}-\mathrm{A} 13 \mathrm{~A}^{\mathrm{iii}}$ & $2.8407(5)$ & $\mathrm{O} 3-\mathrm{A} 11^{\mathrm{xxv}}$ & $1.8799(5)$ \\
\hline $\mathrm{A} 14 \mathrm{~A}-\mathrm{O} 1^{\mathrm{xxi}}$ & $1.9124(6)$ & $\mathrm{O} 3-\mathrm{Mg} 3^{\mathrm{xxv}}$ & $2.0173(7)$ \\
\hline $\mathrm{A} 14 \mathrm{~A}-\mathrm{O} 1$ & $1.9125(6)$ & $\mathrm{O} 4-\mathrm{Cr} 4 \mathrm{~B}^{\mathrm{xxv}}$ & $1.9133(5)$ \\
\hline
\end{tabular}




\begin{tabular}{|c|c|c|c|}
\hline $\mathrm{A} 14 \mathrm{~A}-\mathrm{O} 4^{\mathrm{xxii}}$ & $1.9133(5)$ & $\mathrm{O} 4-\mathrm{A} 14 \mathrm{~A}^{\mathrm{xxv}}$ & $1.9133(5)$ \\
\hline $\mathrm{A} 14 \mathrm{~A}-\mathrm{O} 4^{\mathrm{x}}$ & $1.9133(5)$ & $\mathrm{O} 4-\mathrm{Cr} 4 \mathrm{~B}^{\mathrm{xviii}}$ & $1.9133(5)$ \\
\hline $\mathrm{A} 14 \mathrm{~A}-\mathrm{O} 4^{\mathrm{xxiii}}$ & $1.9133(5)$ & $\mathrm{O} 4-\mathrm{Al} 4 \mathrm{~A}^{\mathrm{xviii}}$ & $1.9133(5)$ \\
\hline $\mathrm{A} 14 \mathrm{~A}-\mathrm{O} 4^{\text {viii }}$ & $1.9133(5)$ & $\mathrm{O} 4-\mathrm{A} 15^{\mathrm{xix}}$ & $1.9136(8)$ \\
\hline $\mathrm{A} 14 \mathrm{~A}-\mathrm{A} 14 \mathrm{~A}^{\mathrm{xiv}}$ & $2.8394(1)$ & $\mathrm{O} 4-\mathrm{Cr} 1 \mathrm{~B}^{\mathrm{xxv}}$ & $1.9295(7)$ \\
\hline $\mathrm{Al} 4 \mathrm{~A}-\mathrm{A} 14 \mathrm{~A}^{\mathrm{xv}}$ & $2.8394(1)$ & $\mathrm{O} 4-\mathrm{Mg} 1 \mathrm{~A}^{\mathrm{xxv}}$ & $1.9295(7)$ \\
\hline $\mathrm{Al} 4 \mathrm{~A}-\mathrm{Cr} 4 \mathrm{~B}^{\mathrm{xiv}}$ & $2.8394(1)$ & $\mathrm{O} 5-\mathrm{A} 15^{\mathrm{xix}}$ & $1.9036(7)$ \\
\hline $\mathrm{Al} 4 \mathrm{~A}-\mathrm{Cr} 4 \mathrm{~B}^{\mathrm{xv}}$ & $2.8394(1)$ & $\mathrm{O} 5-\mathrm{Cr} 3 \mathrm{~B}^{\mathrm{xx}}$ & $1.9188(5)$ \\
\hline $\mathrm{Al} 4 \mathrm{~A}-\mathrm{Al} 4 \mathrm{~A}^{\mathrm{vi}}$ & $2.8394(1)$ & $\mathrm{O} 5-\mathrm{Al} 3 \mathrm{~A}^{\mathrm{xx}}$ & $1.9188(5)$ \\
\hline $\mathrm{Al} 4 \mathrm{~A}-\mathrm{Cr} 4 \mathrm{~B}^{\mathrm{vi}}$ & $2.8394(1)$ & $\mathrm{O} 5-\mathrm{Cr} 2 \mathrm{~B}^{\mathrm{xxv}}$ & $1.9300(7)$ \\
\hline $\mathrm{A} 15-\mathrm{O}^{\mathrm{iii}}$ & $1.9037(7)$ & $\mathrm{O} 5-\mathrm{Mg} 2 \mathrm{~A}^{\mathrm{xxv}}$ & $1.9300(7)$ \\
\hline $\mathrm{A} 15-\mathrm{O} 5^{\mathrm{ii}}$ & $1.9037(7)$ & $\mathrm{O} 6-\mathrm{Cr} 3 \mathrm{~B}^{\mathrm{iii}}$ & $1.8969(6)$ \\
\hline $\mathrm{A} 15-\mathrm{O} 5^{\mathrm{i}}$ & $1.9037(7)$ & $\mathrm{O} 6-\mathrm{A} 13 \mathrm{~A}^{\mathrm{iii}}$ & $1.8969(6)$ \\
\hline $\mathrm{Al} 5-\mathrm{O} 4^{\mathrm{iii}}$ & $1.9136(8)$ & $\mathrm{O} 6-\mathrm{Cr} 3 \mathrm{~B}^{\mathrm{iv}}$ & $1.8969(6)$ \\
\hline $\mathrm{Al} 5-\mathrm{O} 4^{\mathrm{ii}}$ & $1.9136(8)$ & $\mathrm{O} 6-\mathrm{A} 13 \mathrm{~A}^{\mathrm{iv}}$ & $1.8969(6)$ \\
\hline $\mathrm{A} 15-\mathrm{O} 4^{\mathrm{i}}$ & $1.9136(8)$ & $\mathrm{O} 7-\mathrm{Cr} 3 \mathrm{~B}^{\mathrm{ii}}$ & $1.9232(6)$ \\
\hline $\mathrm{A} 15-\mathrm{Cr} 4 \mathrm{~B}^{\mathrm{v}}$ & $2.8515(4)$ & $\mathrm{O} 7-\mathrm{Al}_{3} \mathrm{~A}^{\mathrm{ii}}$ & $1.9232(6)$ \\
\hline $\mathrm{A} 15-\mathrm{A} 14 \mathrm{~A}^{\mathrm{v}}$ & $2.8515(4)$ & $\mathrm{O} 7-\mathrm{Al} 3 \mathrm{~A}^{\mathrm{i}}$ & $1.9232(6)$ \\
\hline $\mathrm{A} 15-\mathrm{Cr} 4 \mathrm{~B}^{\mathrm{iv}}$ & $2.8515(4)$ & $\mathrm{O} 7-\mathrm{Cr} 3 \mathrm{~B}^{\mathrm{i}}$ & $1.9232(6)$ \\
\hline $\mathrm{A} 15-\mathrm{Al}_{4} \mathrm{~A}^{\mathrm{iv}}$ & $2.8515(4)$ & $\mathrm{O} 7-\mathrm{Cr} 3 \mathrm{~B}^{\mathrm{iii}}$ & $1.9232(6)$ \\
\hline $\mathrm{A} 15-\mathrm{Cr} 4 \mathrm{~B}^{\mathrm{vi}}$ & $2.8515(4)$ & $\mathrm{O} 7-\mathrm{A} 13 \mathrm{~A}^{\mathrm{iii}}$ & $1.9232(6)$ \\
\hline $\mathrm{A} 15-\mathrm{A} 14 \mathrm{~A}^{\mathrm{vi}}$ & $2.8515(4)$ & $\mathrm{O} 8-\mathrm{Al} 2^{\mathrm{xix}}$ & $1.7874(7)$ \\
\hline $\mathrm{Mg} 1 \mathrm{~A}-\mathrm{O} 4^{\text {viii }}$ & $1.9295(7)$ & $\mathrm{O} 8-\mathrm{Cr} 3 \mathrm{~B}^{\mathrm{x}}$ & $1.9193(5)$ \\
\hline $\mathrm{Mg} 1 \mathrm{~A}-\mathrm{O} 4^{\mathrm{ii}}$ & $1.9296(7)$ & $\mathrm{O} 8-\mathrm{Al} 3 \mathrm{~A}^{\mathrm{x}}$ & $1.9193(5)$ \\
\hline $\mathrm{Mg} 1 \mathrm{~A}-\mathrm{O} 4^{\mathrm{xvii}}$ & $1.9296(7)$ & $\mathrm{O} 8-\mathrm{Al} 3 \mathrm{~A}^{\mathrm{xiii}}$ & $1.9193(5)$ \\
\hline $\mathrm{Mg} 1 \mathrm{~A}-\mathrm{O} 6$ & $1.9537(12)$ & $\mathrm{O} 8-\mathrm{Cr} 3 \mathrm{~B}^{\mathrm{xiii}}$ & $1.9193(5)$ \\
\hline $\mathrm{Mg} 1 \mathrm{~A}-\mathrm{Al} 5^{\text {xix }}$ & $3.3259(2)$ & $\mathrm{O} 8-\mathrm{Mg} 3^{\mathrm{xiii}}$ & $2.2181(8)$ \\
\hline $\mathrm{O} 7-\mathrm{Be} 1-\mathrm{O}^{\mathrm{i}}$ & $119.45(7)$ & $\mathrm{O} 4^{\mathrm{viii}}-\mathrm{Mg} 1 \mathrm{~A}-\mathrm{O} 4^{\mathrm{ii}}$ & $108.66(2)$ \\
\hline $\mathrm{O} 7-\mathrm{Be} 1-\mathrm{O}^{\mathrm{ii}}$ & $119.45(7)$ & $\mathrm{O} 4^{\mathrm{viii}}-\mathrm{Mg} 1 \mathrm{~A}-\mathrm{O} 4^{\mathrm{xvii}}$ & $108.66(2)$ \\
\hline $\mathrm{O} 3^{\mathrm{i}}-\mathrm{Be} 1-\mathrm{O}^{\mathrm{ii}}$ & $97.89(9)$ & $\mathrm{O} 4^{\mathrm{ii}}-\mathrm{Mg} 1 \mathrm{~A}-\mathrm{O} 4^{\mathrm{xvii}}$ & $108.66(2)$ \\
\hline $\mathrm{O} 7-\mathrm{Be} 1-\mathrm{O} 3^{\mathrm{iii}}$ & $119.45(7)$ & $\mathrm{O} 4^{\text {viii }-\mathrm{Mg} 1 \mathrm{~A}-\mathrm{O} 6}$ & $110.27(2)$ \\
\hline $\mathrm{O} 3^{\mathrm{i}}-\mathrm{Be} 1-\mathrm{O} 3^{\mathrm{iii}}$ & $97.89(9)$ & $\mathrm{O} 4^{\mathrm{ii}}-\mathrm{Mg} 1 \mathrm{~A}-\mathrm{O} 6$ & $110.27(2)$ \\
\hline $\mathrm{O}^{\mathrm{ii}}-\mathrm{Be} 1-\mathrm{O}^{\mathrm{iii}}$ & $97.89(9)$ & $\mathrm{O} 4^{\mathrm{xvii}}-\mathrm{Mg} 1 \mathrm{~A}-\mathrm{O} 6$ & $110.27(2)$ \\
\hline $\mathrm{O} 7-\mathrm{Be} 1-\mathrm{A} 11^{\mathrm{iv}}$ & $138.88(3)$ & 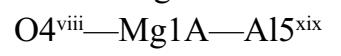 & $121.368(4)$ \\
\hline 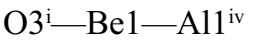 & $48.95(5)$ & $\mathrm{O} 4^{\mathrm{ii}}-\mathrm{Mg} 1 \mathrm{~A}-\mathrm{A} 15^{\mathrm{xix}}$ & $121.369(4)$ \\
\hline $\mathrm{O} 3^{\mathrm{ii}}-\mathrm{Be} 1-\mathrm{A} 11^{\mathrm{iv}}$ & $48.95(5)$ & $\mathrm{O} 4^{\mathrm{xvii}}-\mathrm{Mg} 1 \mathrm{~A}-\mathrm{A} 15^{\mathrm{xix}}$ & $29.94(2)$ \\
\hline $\mathrm{O} 3^{\mathrm{iii}}-\mathrm{Be} 1-\mathrm{A} 11^{\mathrm{iv}}$ & $101.67(10)$ & $\mathrm{O} 6-\mathrm{Mg} 1 \mathrm{~A}-\mathrm{A} 15^{\mathrm{xix}}$ & $80.334(11)$ \\
\hline $\mathrm{O} 7-\mathrm{Be} 1-\mathrm{A} 11^{\mathrm{v}}$ & $138.88(3)$ & $\mathrm{O} 4^{\mathrm{viii}}-\mathrm{Mg} 1 \mathrm{~A}-\mathrm{A} 15$ & $121.368(4)$ \\
\hline $\mathrm{O} 3^{\mathrm{i}}-\mathrm{Be} 1-\mathrm{A} 11^{\mathrm{v}}$ & $48.95(5)$ & $\mathrm{O} 4^{\mathrm{ii}}-\mathrm{Mg} 1 \mathrm{~A}-\mathrm{A} 15$ & $29.94(2)$ \\
\hline $\mathrm{O} 3^{\mathrm{ii}}-\mathrm{Be} 1-\mathrm{Al}^{\mathrm{v}}$ & $101.67(10)$ & $\mathrm{O} 4^{\mathrm{xvii}}-\mathrm{Mg} 1 \mathrm{~A}-\mathrm{A} 15$ & $121.368(4)$ \\
\hline 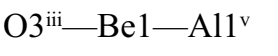 & $48.95(5)$ & $\mathrm{O} 6-\mathrm{Mg} 1 \mathrm{~A}-\mathrm{A} 15$ & $80.336(11)$ \\
\hline $\mathrm{A} 11^{\mathrm{iv}}-\mathrm{Be} 1-\mathrm{A} 11^{\mathrm{v}}$ & $69.44(5)$ & $\mathrm{A} 15^{\mathrm{xix}}-\mathrm{Mg} 1 \mathrm{~A}-\mathrm{A} 15$ & $117.240(6)$ \\
\hline $\mathrm{O} 7-\mathrm{Be} 1-\mathrm{A} 11^{\mathrm{vi}}$ & $138.88(3)$ & $\mathrm{O} 4^{\mathrm{viii}}-\mathrm{Mg} 1 \mathrm{~A}-\mathrm{A} 15^{\mathrm{xiii}}$ & $29.94(2)$ \\
\hline $\mathrm{O} 3^{\mathrm{i}}-\mathrm{Be} 1-\mathrm{A} 11^{\mathrm{vi}}$ & $101.67(10)$ & $\mathrm{O} 4^{\mathrm{ii}}-\mathrm{Mg} 1 \mathrm{~A}-\mathrm{A} 15^{\mathrm{xiii}}$ & $121.369(4)$ \\
\hline 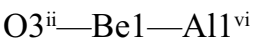 & $48.95(5)$ & $\mathrm{O} 4^{\mathrm{xvii}}-\mathrm{Mg} 1 \mathrm{~A}-\mathrm{A} 15^{\mathrm{xiii}}$ & $121.369(4)$ \\
\hline 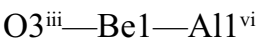 & $48.95(5)$ & $\mathrm{O} 6-\mathrm{Mg} 1 \mathrm{~A}-\mathrm{A} 15^{\mathrm{xiii}}$ & $80.334(11)$ \\
\hline $\mathrm{A} 11^{\mathrm{iv}}-\mathrm{Be} 1-\mathrm{A} 11^{\mathrm{vi}}$ & $69.44(5)$ & $\mathrm{A} 15^{\mathrm{xix}}-\mathrm{Mg} 1 \mathrm{~A}-\mathrm{A} 15^{\text {xiii }}$ & $117.239(6)$ \\
\hline
\end{tabular}




\begin{tabular}{|c|c|}
\hline $\mathrm{A} 11^{\mathrm{v}}-\mathrm{Be} 1-\mathrm{A} 11^{\mathrm{vi}}$ & $69.44(5)$ \\
\hline 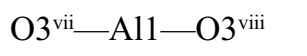 & $180.00(4)$ \\
\hline $\mathrm{O} 3^{\mathrm{vii}}-\mathrm{A} 11-\mathrm{O} 3^{\mathrm{ix}}$ & $83.60(4)$ \\
\hline $\mathrm{O}{ }^{\mathrm{viii}}-\mathrm{A} 11-\mathrm{O} 3^{\text {ix }}$ & $96.40(4)$ \\
\hline $\mathrm{O}^{\text {vii }-\mathrm{A} 11-\mathrm{O}^{\mathrm{x}}}$ & $96.40(4)$ \\
\hline $\mathrm{O} 3^{\text {viii- }}-\mathrm{A} 11-\mathrm{O}^{\mathrm{x}}$ & $83.60(4)$ \\
\hline $\mathrm{O} 3^{\mathrm{ix}}-\mathrm{A} 11-\mathrm{O}^{\mathrm{x}}$ & $180.00(4)$ \\
\hline $\mathrm{O}^{\mathrm{vii}}-\mathrm{A} 11-\mathrm{O} 2$ & $84.58(3)$ \\
\hline $\mathrm{O} 3^{\text {viii_-Al1-O2 }}$ & $95.42(3)$ \\
\hline $\mathrm{O} 3^{\mathrm{ix}}-\mathrm{A} 11-\mathrm{O} 2$ & $84.58(3)$ \\
\hline $\mathrm{O} 3^{\mathrm{x}}-\mathrm{A} 11-\mathrm{O} 2$ & $95.42(3)$ \\
\hline $\mathrm{O} 3^{\mathrm{vii}}-\mathrm{A} 11-\mathrm{O} 2^{\mathrm{xi}}$ & $95.42(3)$ \\
\hline $\mathrm{O}{ }^{\mathrm{viii}}-\mathrm{A} 11-\mathrm{O}^{\mathrm{xi}}$ & $84.58(3)$ \\
\hline $\mathrm{O} 3^{\mathrm{ix}}-\mathrm{A} 11-\mathrm{O} 2^{\mathrm{xi}}$ & $95.42(3)$ \\
\hline $\mathrm{O} 3^{\mathrm{x}}-\mathrm{A} 11-\mathrm{O} 2^{\mathrm{xi}}$ & $84.58(3)$ \\
\hline $\mathrm{O} 2-\mathrm{A} 11-\mathrm{O} 2^{\mathrm{xi}}$ & 180.0 \\
\hline $\mathrm{O}^{\mathrm{vii}}-\mathrm{A} 11-\mathrm{Be}^{\mathrm{xii}}$ & $41.80(2)$ \\
\hline $\mathrm{O} 3^{\mathrm{viii}}-\mathrm{A} 11-\mathrm{Be} 1^{\mathrm{xii}}$ & $138.20(2)$ \\
\hline $\mathrm{O}^{3}{ }^{\mathrm{ix}}-\mathrm{A} 11-\mathrm{Be}^{\mathrm{xii}}$ & $41.80(2)$ \\
\hline $\mathrm{O} 3^{\mathrm{x}}-\mathrm{A} 11-\mathrm{Be}^{\mathrm{xii}}$ & $138.20(2)$ \\
\hline $\mathrm{O} 2-\mathrm{A} 11-\mathrm{Be} 1^{\mathrm{xii}}$ & $82.41(4)$ \\
\hline $\mathrm{O} 2^{\mathrm{xi}}-\mathrm{A} 11-\mathrm{Be} 1^{\mathrm{xii}}$ & $97.59(4)$ \\
\hline $\mathrm{O} 3^{\mathrm{vii}}-\mathrm{A} 11-\mathrm{Be} 1^{\mathrm{xiii}}$ & $138.20(2)$ \\
\hline 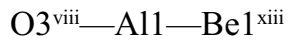 & $41.80(2)$ \\
\hline $\mathrm{O} 3^{\mathrm{ix}}-\mathrm{A} 11-\mathrm{Be} 1^{\mathrm{xiii}}$ & $138.20(2)$ \\
\hline $\mathrm{O} 3^{\mathrm{x}}-\mathrm{A} 11-\mathrm{Be} 1^{\mathrm{xiii}}$ & $41.80(2)$ \\
\hline $\mathrm{O} 2-\mathrm{A} 11-\mathrm{Be} 1^{\mathrm{xiii}}$ & $97.59(4)$ \\
\hline $\mathrm{O} 2^{\mathrm{xi}}-\mathrm{A} 11-\mathrm{Be}^{\mathrm{xiii}}$ & $82.41(4)$ \\
\hline $\mathrm{Be} 1^{\mathrm{xii}}-\mathrm{A} 11-\mathrm{Be} 1^{\mathrm{xiii}}$ & $180.00(7)$ \\
\hline $\mathrm{O}^{\mathrm{vii}}-\mathrm{A} 11-\mathrm{A} 11^{\mathrm{xiv}}$ & $139.043(16)$ \\
\hline $\mathrm{O}^{\mathrm{viii}}-\mathrm{A} 11-\mathrm{A} 11^{\mathrm{xiv}}$ & $40.957(16)$ \\
\hline $\mathrm{O} 3^{\mathrm{ix}}-\mathrm{A} 11-\mathrm{Al} 1^{\mathrm{xiv}}$ & $95.088(18)$ \\
\hline $\mathrm{O}^{\mathrm{x}}-\mathrm{A} 11-\mathrm{Al1}^{\mathrm{xiv}}$ & $84.912(18)$ \\
\hline $\mathrm{O} 2-\mathrm{A} 11-\mathrm{A} 11^{\mathrm{xiv}}$ & $136.211(19)$ \\
\hline $\mathrm{O} 2^{\mathrm{xi}}-\mathrm{A} 11-\mathrm{A} 11^{\mathrm{xiv}}$ & $43.790(19)$ \\
\hline $\mathrm{Be} 1^{\mathrm{xii}}-\mathrm{A} 11-\mathrm{A} 11^{\mathrm{xiv}}$ & $124.72(3)$ \\
\hline $\mathrm{Be} 1^{\mathrm{xiii}}-\mathrm{A} 11-\mathrm{A} 11^{\mathrm{xiv}}$ & $55.28(3)$ \\
\hline $\mathrm{O}^{\mathrm{vii}}-\mathrm{A} 11-\mathrm{Al}^{\mathrm{xv}}$ & $40.957(16)$ \\
\hline $\mathrm{O}^{\mathrm{viii}}-\mathrm{A} 11-\mathrm{A} 11^{\mathrm{xv}}$ & $139.043(16)$ \\
\hline $\mathrm{O} 3^{3 \mathrm{ix}}-\mathrm{A} 11-\mathrm{Al}^{\mathrm{xv}}$ & $84.912(18)$ \\
\hline $\mathrm{O}^{\mathrm{x}}-\mathrm{A} 11-\mathrm{Al}^{\mathrm{xv}}$ & $95.088(18)$ \\
\hline $\mathrm{O} 2-\mathrm{A} 11-\mathrm{A} 11^{\mathrm{xv}}$ & $43.789(19)$ \\
\hline $\mathrm{O} 2^{\mathrm{xi}}-\mathrm{All}-\mathrm{A} 11^{\mathrm{xv}}$ & $136.210(19)$ \\
\hline $\mathrm{Be} 1^{\mathrm{xii}}-\mathrm{A} 11-\mathrm{Al} 1^{\mathrm{xv}}$ & $55.28(3)$ \\
\hline $\mathrm{Be} 1^{\mathrm{xiii}}-\mathrm{A} 11-\mathrm{A} 11^{\mathrm{xv}}$ & $124.72(3)$ \\
\hline $\mathrm{Al1}{ }^{\mathrm{xiv}}-\mathrm{Al} 1-\mathrm{Al1} 1^{\mathrm{xv}}$ & 180.0 \\
\hline $\mathrm{O}^{\mathrm{vii}}-\mathrm{A} 11-\mathrm{A} 11^{\mathrm{vi}}$ & $84.912(18)$ \\
\hline $\mathrm{O} 3^{\text {viii-Al1-Al1 }}{ }^{\mathrm{vi}}$ & $95.088(18)$ \\
\hline
\end{tabular}

\begin{tabular}{|c|c|}
\hline $\mathrm{A} 15-\mathrm{Mg} 1 \mathrm{~A}-\mathrm{A} 15^{\mathrm{xiii}}$ & $117.240(6)$ \\
\hline $\mathrm{O} 4^{\mathrm{viii}}-\mathrm{Mg} 1 \mathrm{~A}-\mathrm{Al} 3 \mathrm{~A}^{\mathrm{iv}}$ & $80.84(2)$ \\
\hline $\mathrm{O} 4^{\mathrm{ii}}-\mathrm{Mg} 1 \mathrm{~A}-\mathrm{A} 13 \mathrm{~A}^{\mathrm{iv}}$ & $122.16(2)$ \\
\hline $\mathrm{O} 4^{\mathrm{xvii}}-\mathrm{Mg} 1 \mathrm{~A}-\mathrm{A} 13 \mathrm{~A}^{\mathrm{iv}}$ & $122.16(2)$ \\
\hline $\mathrm{O} 6-\mathrm{Mg} 1 \mathrm{~A}-\mathrm{Al} 3 \mathrm{~A}^{\mathrm{iv}}$ & $29.432(6)$ \\
\hline $\mathrm{Al} 5^{\mathrm{xix}}-\mathrm{Mg} 1 \mathrm{~A}-\mathrm{A} 13 \mathrm{~A}^{\mathrm{iv}}$ & $95.507(12)$ \\
\hline $\mathrm{Al} 5-\mathrm{Mg} 1 \mathrm{~A}-\mathrm{Al} 3 \mathrm{~A}^{\mathrm{iv}}$ & $95.508(12)$ \\
\hline $\mathrm{Al} 5^{\mathrm{xiii}}-\mathrm{Mg} 1 \mathrm{~A}-\mathrm{Al} 3 \mathrm{~A}^{\mathrm{iv}}$ & $50.903(10)$ \\
\hline 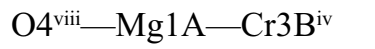 & $80.84(2)$ \\
\hline $\mathrm{O} 4^{\mathrm{ii}}-\mathrm{Mg} 1 \mathrm{~A}-\mathrm{Cr} 3 \mathrm{~B}^{\mathrm{iv}}$ & $122.16(2)$ \\
\hline $\mathrm{O} 4^{\mathrm{xvi}}-\mathrm{Mg} 1 \mathrm{~A}-\mathrm{Cr} \mathrm{B}^{\mathrm{iv}}$ & $122.16(2)$ \\
\hline $\mathrm{O} 6-\mathrm{Mg} 1 \mathrm{~A}-\mathrm{Cr} 3 \mathrm{~B}^{\mathrm{iv}}$ & $29.432(6)$ \\
\hline $\mathrm{A} 15^{\mathrm{xix}}-\mathrm{Mg} 1 \mathrm{~A}-\mathrm{Cr} 3 \mathrm{~B}^{\mathrm{iv}}$ & $95.507(12)$ \\
\hline $\mathrm{Al} 5-\mathrm{Mg} 1 \mathrm{~A}-\mathrm{Cr} 3 \mathrm{~B}^{\mathrm{iv}}$ & $95.508(12)$ \\
\hline $\mathrm{Al} 5^{\mathrm{xiii}}-\mathrm{Mg} 1 \mathrm{~A}-\mathrm{Cr} 3 \mathrm{~B}^{\mathrm{iv}}$ & $50.903(10)$ \\
\hline $\mathrm{A} 13 \mathrm{~A}^{\mathrm{iv}}-\mathrm{Mg} 1 \mathrm{~A}-\mathrm{Cr} 3 \mathrm{~B}^{\mathrm{iv}}$ & $0.000(5)$ \\
\hline $\mathrm{O} 4^{\mathrm{viii}}-\mathrm{Mg} 1 \mathrm{~A}-\mathrm{Al} 3 \mathrm{~A}^{\mathrm{iii}}$ & $122.16(2)$ \\
\hline $\mathrm{O} 4^{\mathrm{ii}}-\mathrm{Mg} 1 \mathrm{~A}-\mathrm{A} 13 \mathrm{~A}^{\mathrm{iii}}$ & $80.84(2)$ \\
\hline $\mathrm{O} 4^{\mathrm{xvi}}-\mathrm{Mg} 1 \mathrm{~A}-\mathrm{A} 13 \mathrm{~A}^{\mathrm{iii}}$ & $122.16(2)$ \\
\hline $\mathrm{O} 6-\mathrm{Mg} 1 \mathrm{~A}-\mathrm{A} 13 \mathrm{~A}^{\mathrm{iii}}$ & $29.432(6)$ \\
\hline $\mathrm{A} 15^{\mathrm{xix}}-\mathrm{Mg} 1 \mathrm{~A}-\mathrm{A} 13 \mathrm{~A}^{\mathrm{iii}}$ & $95.508(12)$ \\
\hline $\mathrm{Al} 5-\mathrm{Mg} 1 \mathrm{~A}-\mathrm{Al} 3 \mathrm{~A}^{\mathrm{iii}}$ & $50.904(10)$ \\
\hline $\mathrm{A} 15^{\mathrm{xiii}}-\mathrm{Mg} 1 \mathrm{~A}-\mathrm{A} 13 \mathrm{~A}^{\mathrm{iii}}$ & $95.508(12)$ \\
\hline $\mathrm{A} 13 \mathrm{~A}^{\mathrm{iv}}-\mathrm{Mg} 1 \mathrm{~A}-\mathrm{A} 13 \mathrm{~A}^{\mathrm{iii}}$ & $50.371(11)$ \\
\hline $\mathrm{Cr} 3 \mathrm{~B}^{\mathrm{iv}}-\mathrm{Mg} 1 \mathrm{~A}-\mathrm{A} 13 \mathrm{~A}^{\mathrm{iii}}$ & 50.4 \\
\hline $\mathrm{O} 4^{\mathrm{viii}}-\mathrm{Mg} 1 \mathrm{~A}-\mathrm{Cr} 3 \mathrm{~B}^{\mathrm{iii}}$ & $122.16(2)$ \\
\hline $\mathrm{O} 4^{\mathrm{ii}}-\mathrm{Mg} 1 \mathrm{~A}-\mathrm{Cr} 3 \mathrm{~B}^{\mathrm{iii}}$ & $80.84(2)$ \\
\hline $\mathrm{O} 4^{\mathrm{xvii}}-\mathrm{Mg} 1 \mathrm{~A}-\mathrm{Cr} 3 \mathrm{~B}^{\mathrm{iii}}$ & $122.16(2)$ \\
\hline $\mathrm{O} 6-\mathrm{Mg} 1 \mathrm{~A}-\mathrm{Cr} 3 \mathrm{~B}^{\mathrm{iii}}$ & $29.432(6)$ \\
\hline $\mathrm{A} 15^{\mathrm{xix}}-\mathrm{Mg} 1 \mathrm{~A}-\mathrm{Cr} 3 \mathrm{~B}^{\mathrm{iii}}$ & $95.508(12)$ \\
\hline $\mathrm{Al} 5-\mathrm{Mg} 1 \mathrm{~A}-\mathrm{Cr} 3 \mathrm{~B}^{\mathrm{iii}}$ & $50.904(10)$ \\
\hline $\mathrm{A} 15^{\mathrm{xiii}}-\mathrm{Mg} 1 \mathrm{~A}-\mathrm{Cr} 3 \mathrm{~B}^{\mathrm{iii}}$ & $95.508(12)$ \\
\hline $\mathrm{A} 13 \mathrm{~A}^{\mathrm{iv}}-\mathrm{Mg} 1 \mathrm{~A}-\mathrm{Cr} 3 \mathrm{~B}^{\mathrm{iii}}$ & $50.371(11)$ \\
\hline $\mathrm{Cr} 3 \mathrm{~B}^{\mathrm{iv}}-\mathrm{Mg} 1 \mathrm{~A}-\mathrm{Cr} 3 \mathrm{~B}^{\mathrm{iii}}$ & $50.371(11)$ \\
\hline $\mathrm{A} 13 \mathrm{~A}^{\mathrm{iii}}-\mathrm{Mg} 1 \mathrm{~A}-\mathrm{Cr} 3 \mathrm{~B}^{\mathrm{iii}}$ & $0.000(15)$ \\
\hline $\mathrm{O} 5^{\text {viii }}-\mathrm{Mg} 2 \mathrm{~A}-\mathrm{O}^{\text {xxiv }}$ & $109.66(2)$ \\
\hline 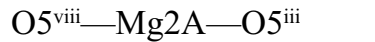 & $109.66(2)$ \\
\hline $\mathrm{O}^{\mathrm{xxiv}}-\mathrm{Mg} 2 \mathrm{~A}-\mathrm{O} 5^{\mathrm{iii}}$ & $109.66(2)$ \\
\hline $\mathrm{O}^{\mathrm{viii}}-\mathrm{Mg} 2 \mathrm{~A}-\mathrm{O} 1$ & $109.28(2)$ \\
\hline $\mathrm{O} 5^{\mathrm{xxiv}}-\mathrm{Mg} 2 \mathrm{~A}-\mathrm{O} 1$ & $109.28(2)$ \\
\hline $\mathrm{O} 5^{\mathrm{iii}}-\mathrm{Mg} 2 \mathrm{~A}-\mathrm{O} 1$ & $109.28(2)$ \\
\hline $\mathrm{O} 5^{\mathrm{viii}}-\mathrm{Mg} 2 \mathrm{~A}-\mathrm{A} 15^{\mathrm{viii}}$ & $121.522(3)$ \\
\hline $\mathrm{O} 5^{\text {xiv }}-\mathrm{Mg} 2 \mathrm{~A}-\mathrm{A} 15^{\text {viii }}$ & $29.45(2)$ \\
\hline $\mathrm{O} 5^{\mathrm{iii}}-\mathrm{Mg} 2 \mathrm{~A}-\mathrm{A} 15^{\mathrm{viii}}$ & $121.522(3)$ \\
\hline $\mathrm{O} 1-\mathrm{Mg} 2 \mathrm{~A}-\mathrm{A} 15^{\text {viii }}$ & $79.835(12)$ \\
\hline $\mathrm{O} 5^{\mathrm{viii}}-\mathrm{Mg} 2 \mathrm{~A}-\mathrm{A} 15^{\mathrm{xiii}}$ & $29.45(2)$ \\
\hline $\mathrm{O} 5^{\mathrm{xxi}}-\mathrm{Mg} 2 \mathrm{~A}-\mathrm{A} 15^{\mathrm{xiii}}$ & $121.522(3)$ \\
\hline 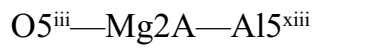 & $121.522(3)$ \\
\hline
\end{tabular}




\begin{tabular}{|c|c|}
\hline $\mathrm{O}^{\mathrm{ix}}-\mathrm{A} 11-\mathrm{A} 11^{\mathrm{vi}}$ & $40.957(16)$ \\
\hline $\mathrm{O} 3^{\mathrm{x}}-\mathrm{A} 11-\mathrm{A} 11^{\mathrm{vi}}$ & $139.043(16)$ \\
\hline $\mathrm{O} 2-\mathrm{A} 11-\mathrm{A} 11^{\mathrm{vi}}$ & $43.789(19)$ \\
\hline $\mathrm{O} 2^{\mathrm{xi}}-\mathrm{A} 11-\mathrm{A} 11^{\mathrm{vi}}$ & $136.211(19)$ \\
\hline $\mathrm{Be} 1^{\mathrm{xi}}-\mathrm{A} 11-\mathrm{A} 11^{\mathrm{vi}}$ & $55.28(3)$ \\
\hline $\mathrm{Be} 1^{\mathrm{xiii}}-\mathrm{A} 11-\mathrm{Al} 1^{\mathrm{vi}}$ & $124.72(3)$ \\
\hline $\mathrm{A} 11^{\mathrm{xiv}}-\mathrm{A} 11-\mathrm{A} 11^{\mathrm{vi}}$ & 120.0 \\
\hline $\mathrm{All} 1^{\mathrm{xv}}-\mathrm{A} 11-\mathrm{Al} 1^{\mathrm{vi}}$ & 60.0 \\
\hline $\mathrm{O}^{\mathrm{vii}}-\mathrm{A} 11-\mathrm{A} 11^{\mathrm{xvi}}$ & $95.088(18)$ \\
\hline 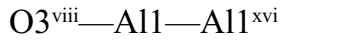 & $84.912(18)$ \\
\hline $\mathrm{O} 3^{\mathrm{ix}}-\mathrm{A} 11-\mathrm{A} 11^{\mathrm{xvi}}$ & $139.043(16)$ \\
\hline $\mathrm{O}^{\mathrm{x}}-\mathrm{A} 11-\mathrm{A} 11^{\mathrm{xvi}}$ & $40.957(16)$ \\
\hline $\mathrm{O} 2-\mathrm{A} 11-\mathrm{A} 11^{\mathrm{xvi}}$ & $136.211(19)$ \\
\hline $\mathrm{O} 2^{\mathrm{xi}}-\mathrm{A} 11-\mathrm{A} 11^{\mathrm{xvi}}$ & $43.789(19)$ \\
\hline $\mathrm{Be}^{\mathrm{xii}}-\mathrm{A} 11-\mathrm{Al} 1^{\mathrm{xvi}}$ & $124.72(3)$ \\
\hline $\mathrm{Be} 1^{\mathrm{xiii}}-\mathrm{A} 11-\mathrm{Al} 1^{\mathrm{xvi}}$ & $55.28(3)$ \\
\hline $\mathrm{All1}{ }^{\mathrm{xiv}}-\mathrm{A} 11-\mathrm{A} 11^{\mathrm{xvi}}$ & 60.0 \\
\hline $\mathrm{Al1} 1^{\mathrm{xv}}-\mathrm{Al} 1-\mathrm{Al1} 1^{\mathrm{xvi}}$ & 120.0 \\
\hline $\mathrm{A} 11^{\mathrm{vi}}-\mathrm{A} 11-\mathrm{A} 11^{\mathrm{xvi}}$ & 180.0 \\
\hline $\mathrm{O} 2-\mathrm{A} 12-\mathrm{O} 8^{\mathrm{i}}$ & $109.22(3)$ \\
\hline $\mathrm{O} 2-\mathrm{A} 12-\mathrm{O}^{\mathrm{iv}}$ & $109.22(3)$ \\
\hline $\mathrm{O} 8^{\mathrm{i}}-\mathrm{A} 12-\mathrm{O}^{\mathrm{iv}}$ & $109.72(3)$ \\
\hline $\mathrm{O} 2-\mathrm{A} 12-\mathrm{O}^{\mathrm{xvi}}$ & $109.22(3)$ \\
\hline $\mathrm{O} 8^{\mathrm{i}}-\mathrm{A} 12-\mathrm{O}^{\mathrm{xvi}}$ & $109.72(3)$ \\
\hline $\mathrm{O}^{\mathrm{iv}}-\mathrm{A} 12-\mathrm{O}^{\mathrm{xvi}}$ & $109.72(3)$ \\
\hline $\mathrm{O} 6-\mathrm{Al} 3 \mathrm{~A}-\mathrm{O} 5^{\mathrm{xvii}}$ & $96.66(3)$ \\
\hline $\mathrm{O} 6-\mathrm{A} 13 \mathrm{~A}-\mathrm{O} 5$ & $96.66(3)$ \\
\hline $\mathrm{O} 5^{\mathrm{xvii}}-\mathrm{A} 13 \mathrm{~A}-\mathrm{O} 5$ & $82.22(4)$ \\
\hline $\mathrm{O} 6-\mathrm{Al} 3 \mathrm{~A}-\mathrm{O}^{\mathrm{v}}$ & $83.72(3)$ \\
\hline $\mathrm{O}^{\mathrm{xvii}}-\mathrm{Al} 3 \mathrm{~A}-\mathrm{O}^{\mathrm{v}}$ & $175.23(3)$ \\
\hline $\mathrm{O} 5-\mathrm{A} 13 \mathrm{~A}-\mathrm{O}^{\mathrm{v}}$ & $93.01(3)$ \\
\hline $\mathrm{O} 6-\mathrm{A} 13 \mathrm{~A}-\mathrm{O} 8^{\mathrm{xviii}}$ & $83.72(3)$ \\
\hline $\mathrm{O} 5^{\mathrm{xvi}}-\mathrm{Al} 3 \mathrm{~A}-\mathrm{O} 8^{\mathrm{xviii}}$ & $93.01(3)$ \\
\hline $\mathrm{O} 5-\mathrm{Al} 3 \mathrm{~A}-\mathrm{O} 8^{\mathrm{xviii}}$ & $175.23(3)$ \\
\hline $\mathrm{O} 8^{\mathrm{v}}-\mathrm{A} 13 \mathrm{~A}-\mathrm{O} 8^{\mathrm{xviii}}$ & $91.76(4)$ \\
\hline $\mathrm{O} 6-\mathrm{A} 13 \mathrm{~A}-\mathrm{O} 7^{\mathrm{xix}}$ & $178.60(4)$ \\
\hline $\mathrm{O} 5^{\mathrm{xvii}}-\mathrm{Al} 3 \mathrm{~A}-\mathrm{O} 7^{\mathrm{xix}}$ & $84.39(3)$ \\
\hline $\mathrm{O} 5-\mathrm{Al} 3 \mathrm{~A}-\mathrm{O} 7^{\mathrm{xix}}$ & $84.40(3)$ \\
\hline $\mathrm{O} 8^{\mathrm{v}}-\mathrm{A} 13 \mathrm{~A}-\mathrm{O} 7^{\mathrm{xix}}$ & $95.31(3)$ \\
\hline $\mathrm{O} 8^{\mathrm{xviii}}-\mathrm{Al} 3 \mathrm{~A}-\mathrm{O} 7^{\mathrm{xix}}$ & $95.31(3)$ \\
\hline $\mathrm{O} 6-\mathrm{A} 13 \mathrm{~A}-\mathrm{Cr} 3 \mathrm{~B}^{\mathrm{xvii}}$ & $138.48(2)$ \\
\hline $\mathrm{O}^{\mathrm{xvii}}-\mathrm{Al} 3 \mathrm{~A}-\mathrm{Cr} 3 \mathrm{~B}^{\mathrm{xvii}}$ & $42.307(16)$ \\
\hline $\mathrm{O} 5-\mathrm{Al} 3 \mathrm{~A}-\mathrm{Cr} 3 \mathrm{~B}^{\mathrm{xvii}}$ & $85.296(19)$ \\
\hline $\mathrm{O} 8^{\mathrm{v}}-\mathrm{A} 13 \mathrm{~A}-\mathrm{Cr} 3 \mathrm{~B}^{\mathrm{xvii}}$ & $137.735(18)$ \\
\hline $\mathrm{O} 8^{\mathrm{xviii}}-\mathrm{A} 13 \mathrm{~A}-\mathrm{Cr} 3 \mathrm{~B}^{\mathrm{xvii}}$ & $91.271(18)$ \\
\hline $\mathrm{O} 7^{\mathrm{xix}}-\mathrm{A} 13 \mathrm{~A}-\mathrm{Cr} 3 \mathrm{~B}^{\mathrm{xvii}}$ & $42.450(19)$ \\
\hline $\mathrm{O} 6-\mathrm{Al} 3 \mathrm{~A}-\mathrm{Al} 3 \mathrm{~A}^{\mathrm{xvii}}$ & $138.48(2)$ \\
\hline $\mathrm{O} 5^{\mathrm{xvi}}-\mathrm{A} 13 \mathrm{~A}-\mathrm{A} 13 \mathrm{~A}^{\mathrm{xvii}}$ & $42.307(16)$ \\
\hline
\end{tabular}

\begin{tabular}{|c|c|}
\hline $\mathrm{O} 1-\mathrm{Mg} 2 \mathrm{~A}-\mathrm{A} 15^{\mathrm{xiii}}$ & $79.835(12)$ \\
\hline $\mathrm{A} 15^{\mathrm{viii}}-\mathrm{Mg} 2 \mathrm{~A}-\mathrm{A} 15^{\mathrm{xiii}}$ & $116.954(7)$ \\
\hline $\mathrm{O}^{\mathrm{viii}-\mathrm{Mg} 2 \mathrm{~A}-\mathrm{A} 15}$ & $121.521(4)$ \\
\hline $\mathrm{O}^{\mathrm{xxiv}}-\mathrm{Mg} 2 \mathrm{~A}-\mathrm{A} 15$ & $121.521(3)$ \\
\hline $\mathrm{O}^{\mathrm{iii}}-\mathrm{Mg} 2 \mathrm{~A}-\mathrm{A} 15$ & $29.45(2)$ \\
\hline $\mathrm{O} 1-\mathrm{Mg} 2 \mathrm{~A}-\mathrm{Al} 5$ & $79.834(12)$ \\
\hline 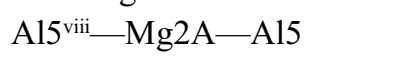 & $116.954(7)$ \\
\hline $\mathrm{A} 15^{\mathrm{xiii}}-\mathrm{Mg} 2 \mathrm{~A}-\mathrm{A} 15$ & $116.954(7)$ \\
\hline 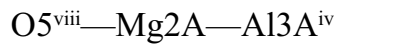 & $29.673(12)$ \\
\hline $\mathrm{O}^{\mathrm{xxiv}}-\mathrm{Mg} 2 \mathrm{~A}-\mathrm{A} 13 \mathrm{~A}^{\mathrm{iv}}$ & $121.37(3)$ \\
\hline $\mathrm{O}^{\mathrm{iii}}-\mathrm{Mg} 2 \mathrm{~A}-\mathrm{Al} 3 \mathrm{~A}^{\mathrm{iv}}$ & $79.990(15)$ \\
\hline $\mathrm{O} 1-\mathrm{Mg} 2 \mathrm{~A}-\mathrm{A} 13 \mathrm{~A}^{\mathrm{iv}}$ & $121.802(9)$ \\
\hline $\mathrm{Al} 5^{\mathrm{viii}}-\mathrm{Mg} 2 \mathrm{~A}-\mathrm{Al} 3 \mathrm{~A}^{\mathrm{iv}}$ & $144.821(12)$ \\
\hline $\mathrm{Al} 5^{\mathrm{xii}}-\mathrm{Mg} 2 \mathrm{~A}-\mathrm{Al} 3 \mathrm{~A}^{\mathrm{iv}}$ & $50.834(9)$ \\
\hline $\mathrm{Al} 5-\mathrm{Mg} 2 \mathrm{~A}-\mathrm{Al} 3 \mathrm{~A}^{\mathrm{iv}}$ & $95.349(7)$ \\
\hline $\mathrm{O}^{\text {viii- }} \mathrm{Mg} 2 \mathrm{~A}-\mathrm{Al} 3 \mathrm{~A}^{\text {viii }}$ & $29.674(12)$ \\
\hline $\mathrm{O}^{\text {xxiv }}-\mathrm{Mg} 2 \mathrm{~A}-\mathrm{Al} 3 \mathrm{~A}^{\text {viii }}$ & $79.990(15)$ \\
\hline $\mathrm{O}^{\mathrm{iii}}-\mathrm{Mg} 2 \mathrm{~A}-\mathrm{Al} 3 \mathrm{~A}^{\mathrm{viii}}$ & $121.37(3)$ \\
\hline $\mathrm{O} 1-\mathrm{Mg} 2 \mathrm{~A}-\mathrm{Al} 3 \mathrm{~A}^{\mathrm{viii}}$ & $121.802(9)$ \\
\hline $\mathrm{Al} 5^{\text {viii }-M g 2 A}-\mathrm{Al} 3 \mathrm{~A}^{\text {viii }}$ & $95.348(7)$ \\
\hline $\mathrm{Al} 5^{\text {xiii }-M g 2 A}-\mathrm{Al} 3 \mathrm{~A}^{\mathrm{viii}}$ & $50.834(8)$ \\
\hline $\mathrm{Al} 5-\mathrm{Mg} 2 \mathrm{~A}-\mathrm{Al} 3 \mathrm{~A}^{\mathrm{viii}}$ & $144.822(12)$ \\
\hline $\mathrm{A} 13 \mathrm{~A}^{\mathrm{iv}}-\mathrm{Mg} 2 \mathrm{~A}-\mathrm{Al} 3 \mathrm{~A}^{\mathrm{viii}}$ & $50.270(9)$ \\
\hline 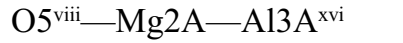 & $79.990(15)$ \\
\hline $\mathrm{O}^{\mathrm{xxiv}}-\mathrm{Mg} 2 \mathrm{~A}-\mathrm{A} 13 \mathrm{~A}^{\mathrm{xvi}}$ & $29.674(12)$ \\
\hline $\mathrm{O}^{\mathrm{iii}}-\mathrm{Mg} 2 \mathrm{~A}-\mathrm{A} 13 \mathrm{~A}^{\mathrm{xvi}}$ & $121.37(3)$ \\
\hline $\mathrm{O} 1-\mathrm{Mg} 2 \mathrm{~A}-\mathrm{A} 13 \mathrm{~A}^{\mathrm{xvi}}$ & $121.802(9)$ \\
\hline 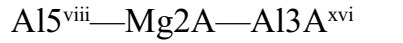 & $50.834(9)$ \\
\hline $\mathrm{A} 15^{\mathrm{xiii}}-\mathrm{Mg} 2 \mathrm{~A}-\mathrm{Al} 3 \mathrm{~A}^{\mathrm{xvi}}$ & $95.348(7)$ \\
\hline $\mathrm{A} 15-\mathrm{Mg} 2 \mathrm{~A}-\mathrm{Al} 3 \mathrm{~A}^{\mathrm{xvi}}$ & $144.822(12)$ \\
\hline $\mathrm{A} 13 \mathrm{~A}^{\mathrm{iv}}-\mathrm{Mg} 2 \mathrm{~A}-\mathrm{Al} 3 \mathrm{~A}^{\mathrm{xvi}}$ & $94.786(12)$ \\
\hline $\mathrm{A} 13 \mathrm{~A}^{\mathrm{viii}}-\mathrm{Mg} 2 \mathrm{~A}-\mathrm{Al} 3 \mathrm{~A}^{\mathrm{xvi}}$ & $50.318(10)$ \\
\hline 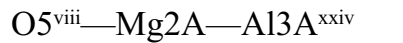 & $121.37(3)$ \\
\hline $\mathrm{O}^{\mathrm{xxiv}}-\mathrm{Mg} 2 \mathrm{~A}-\mathrm{A} 13 \mathrm{~A}^{\mathrm{xxiv}}$ & $29.673(12)$ \\
\hline $\mathrm{O}^{\mathrm{iii}}-\mathrm{Mg} 2 \mathrm{~A}-\mathrm{A} 13 \mathrm{~A}^{\mathrm{xxiv}}$ & $79.990(15)$ \\
\hline $\mathrm{O} 1-\mathrm{Mg} 2 \mathrm{~A}-\mathrm{A} 13 \mathrm{~A}^{\mathrm{xxiv}}$ & $121.802(9)$ \\
\hline $\mathrm{A} 15^{\text {viii }}-\mathrm{Mg} 2 \mathrm{~A}-\mathrm{Al} 3 \mathrm{~A}^{\mathrm{xxiv}}$ & $50.834(8)$ \\
\hline $\mathrm{A} 15^{\mathrm{xii}}-\mathrm{Mg} 2 \mathrm{~A}-\mathrm{A} 13 \mathrm{~A}^{\mathrm{xxiv}}$ & $144.821(12)$ \\
\hline $\mathrm{A} 15-\mathrm{Mg} 2 \mathrm{~A}-\mathrm{A} 13 \mathrm{~A}^{\mathrm{xxiv}}$ & $95.349(7)$ \\
\hline $\mathrm{A} 13 \mathrm{~A}^{\mathrm{iv}}-\mathrm{Mg} 2 \mathrm{~A}-\mathrm{A} 13 \mathrm{~A}^{\mathrm{xxiv}}$ & $116.397(17)$ \\
\hline $\mathrm{A} 13 \mathrm{~A}^{\mathrm{viii}}-\mathrm{Mg} 2 \mathrm{~A}-\mathrm{A} 13 \mathrm{~A}^{\mathrm{xxiv}}$ & $94.786(12)$ \\
\hline $\mathrm{A} 13 \mathrm{~A}^{\mathrm{xvi}}-\mathrm{Mg} 2 \mathrm{~A}-\mathrm{A} 13 \mathrm{~A}^{\mathrm{xxiv}}$ & $50.270(9)$ \\
\hline $\mathrm{O} 5^{\mathrm{viii}}-\mathrm{Mg} 2 \mathrm{~A}-\mathrm{A} 13 \mathrm{~A}^{\mathrm{iii}}$ & $79.989(15)$ \\
\hline $\mathrm{O}^{\mathrm{xxiv}}-\mathrm{Mg} 2 \mathrm{~A}-\mathrm{Al} 3 \mathrm{~A}^{\mathrm{iii}}$ & $121.37(3)$ \\
\hline $\mathrm{O}^{\mathrm{iii}}-\mathrm{Mg} 2 \mathrm{~A}-\mathrm{A} 13 \mathrm{~A}^{\mathrm{iii}}$ & $29.674(12)$ \\
\hline $\mathrm{O} 1-\mathrm{Mg} 2 \mathrm{~A}-\mathrm{A} 13 \mathrm{~A}^{\mathrm{iii}}$ & $121.802(9)$ \\
\hline $\mathrm{Al} 5^{\mathrm{viii}}-\mathrm{Mg} 2 \mathrm{~A}-\mathrm{Al} 3 \mathrm{~A}^{\mathrm{iii}}$ & $144.822(12)$ \\
\hline $\mathrm{A} 15^{\mathrm{xiii}}-\mathrm{Mg} 2 \mathrm{~A}-\mathrm{A} 13 \mathrm{~A}^{\mathrm{iii}}$ & $95.349(7)$ \\
\hline
\end{tabular}




\begin{tabular}{|c|c|c|c|}
\hline $\mathrm{O} 5-\mathrm{Al} 3 \mathrm{~A}-\mathrm{Al} 3 \mathrm{~A}^{\mathrm{xvii}}$ & $85.296(19)$ & $\mathrm{A} 15-\mathrm{Mg} 2 \mathrm{~A}-\mathrm{A} 13 \mathrm{~A}^{\mathrm{iii}}$ & $50.835(9)$ \\
\hline $\mathrm{O} 8^{\mathrm{v}}-\mathrm{A} 13 \mathrm{~A}-\mathrm{A} 13 \mathrm{~A}^{\mathrm{xvii}}$ & $137.735(18)$ & $\mathrm{A} 13 \mathrm{~A}^{\mathrm{iv}}-\mathrm{Mg} 2 \mathrm{~A}-\mathrm{A} 13 \mathrm{~A}^{\mathrm{iii}}$ & $50.317(10)$ \\
\hline $\mathrm{O} 8^{\mathrm{xvii}}-\mathrm{Al} 3 \mathrm{~A}-\mathrm{Al} 3 \mathrm{~A}^{\mathrm{xvii}}$ & $91.271(18)$ & $\mathrm{A} 13 \mathrm{~A}^{\mathrm{viii}}-\mathrm{Mg} 2 \mathrm{~A}-\mathrm{A} 13 \mathrm{~A}^{\mathrm{iii}}$ & $94.786(12)$ \\
\hline $\mathrm{O}^{\mathrm{xix}}-\mathrm{Al} 3 \mathrm{~A}-\mathrm{Al} 3 \mathrm{~A}^{\mathrm{xvii}}$ & $42.450(19)$ & $\mathrm{Al} 3 \mathrm{~A}^{\mathrm{xvi}}-\mathrm{Mg} 2 \mathrm{~A}-\mathrm{Al} 3 \mathrm{~A}^{\mathrm{iii}}$ & $116.397(17)$ \\
\hline $\mathrm{Cr} 3 \mathrm{~B}^{\mathrm{xvii}}-\mathrm{Al} 3 \mathrm{~A}-\mathrm{A} 13 \mathrm{~A}^{\mathrm{xvii}}$ & 0.0 & $\mathrm{~A} 13 \mathrm{~A}^{\mathrm{xxiv}}-\mathrm{Mg} 2 \mathrm{~A}-\mathrm{A} 13 \mathrm{~A}^{\mathrm{iii}}$ & $94.786(12)$ \\
\hline $\mathrm{O} 6-\mathrm{A} 13 \mathrm{~A}-\mathrm{Al} 3 \mathrm{~A}^{\mathrm{xx}}$ & $138.48(2)$ & 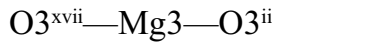 & $103.70(3)$ \\
\hline $\mathrm{O}^{\mathrm{xvii}}-\mathrm{A} 13 \mathrm{~A}-\mathrm{A} 13 \mathrm{~A}^{\mathrm{xx}}$ & $85.296(19)$ & $\mathrm{O} 3^{\mathrm{xvii}}-\mathrm{Mg} 3-\mathrm{O} 3^{\text {viii }}$ & $103.70(3)$ \\
\hline $\mathrm{O} 5-\mathrm{A} 13 \mathrm{~A}-\mathrm{A} 13 \mathrm{~A}^{\mathrm{xx}}$ & $42.308(16)$ & $\mathrm{O} 3^{\mathrm{ii}}-\mathrm{Mg} 3-\mathrm{O} 3^{\mathrm{viii}}$ & $103.70(3)$ \\
\hline $\mathrm{O} 8^{\mathrm{v}}-\mathrm{Al} 3 \mathrm{~A}-\mathrm{Al} 3 \mathrm{~A}^{\mathrm{xx}}$ & $91.271(18)$ & $\mathrm{O} 3^{\mathrm{xvii}}-\mathrm{Mg} 3-\mathrm{O} 8^{\mathrm{xvi}}$ & $160.59(4)$ \\
\hline $\mathrm{O} 8^{\mathrm{xviii}}-\mathrm{A} 13 \mathrm{~A}-\mathrm{Al} 3 \mathrm{~A}^{\mathrm{xx}}$ & $137.735(18)$ & $\mathrm{O} 3^{\mathrm{ii}}-\mathrm{Mg} 3-\mathrm{O} 8^{\mathrm{xvi}}$ & $88.06(2)$ \\
\hline $\mathrm{O} 7^{\mathrm{xix}}-\mathrm{Al} 3 \mathrm{~A}-\mathrm{Al} 3 \mathrm{~A}^{\mathrm{xx}}$ & $42.451(19)$ & 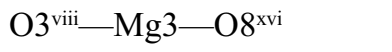 & $88.06(2)$ \\
\hline $\mathrm{Cr} 3 \mathrm{~B}^{\mathrm{xvii}}-\mathrm{A} 13 \mathrm{~A}-\mathrm{Al} 3 \mathrm{~A}^{\mathrm{xx}}$ & 60.0 & $\mathrm{O} 3^{\mathrm{xvii}}-\mathrm{Mg} 3-\mathrm{O}^{\mathrm{v}}$ & $88.06(2)$ \\
\hline $\mathrm{Al} 3 \mathrm{~A}^{\mathrm{xvii}}-\mathrm{Al} 3 \mathrm{~A}-\mathrm{Al} 3 \mathrm{~A}^{\mathrm{xx}}$ & 60.0 & $\mathrm{O} 3^{\mathrm{ii}}-\mathrm{Mg} 3-\mathrm{O}^{\mathrm{v}}$ & $88.06(2)$ \\
\hline $\mathrm{O} 6-\mathrm{Al} 3 \mathrm{~A}-\mathrm{Cr} 3 \mathrm{~B}^{\mathrm{xx}}$ & $138.48(2)$ & 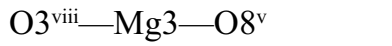 & $160.59(4)$ \\
\hline $\mathrm{O} 5^{\mathrm{xvii}}-\mathrm{A} 13 \mathrm{~A}-\mathrm{Cr} 3 \mathrm{~B}^{\mathrm{xx}}$ & $85.296(19)$ & $\mathrm{O} 8^{\mathrm{xvi}}-\mathrm{Mg} 3-\mathrm{O}^{\mathrm{v}}$ & $76.80(3)$ \\
\hline $\mathrm{O} 5-\mathrm{A} 13 \mathrm{~A}-\mathrm{Cr} 3 \mathrm{~B}^{\mathrm{xx}}$ & $42.308(16)$ & $\mathrm{O} 3^{\mathrm{xvii}}-\mathrm{Mg} 3-\mathrm{O} 8^{\mathrm{xviii}}$ & 88.064 (19) \\
\hline $\mathrm{O} 8^{\mathrm{v}}-\mathrm{A} 13 \mathrm{~A}-\mathrm{Cr} 3 \mathrm{~B}^{\mathrm{xx}}$ & $91.271(18)$ & $\mathrm{O} 3^{\mathrm{ii}}-\mathrm{Mg} 3-\mathrm{O} 8^{\mathrm{xviii}}$ & $160.59(4)$ \\
\hline $\mathrm{O} 8^{\mathrm{xviii}}-\mathrm{A} 13 \mathrm{~A}-\mathrm{Cr} 3 \mathrm{~B}^{\mathrm{xx}}$ & $137.735(18)$ & 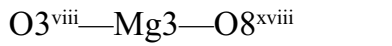 & $88.06(2)$ \\
\hline $\mathrm{O}^{\mathrm{xix}}-\mathrm{A} 13 \mathrm{~A}-\mathrm{Cr} 3 \mathrm{~B}^{\mathrm{xx}}$ & $42.451(19)$ & $\mathrm{O} 8^{\mathrm{xvi}}-\mathrm{Mg} 3-\mathrm{O} 8^{\mathrm{xvii}}$ & $76.80(3)$ \\
\hline $\mathrm{Cr} 3 \mathrm{~B}^{\mathrm{xvii}}-\mathrm{A} 13 \mathrm{~A}-\mathrm{Cr} 3 \mathrm{~B}^{\mathrm{xx}}$ & 60.0 & $\mathrm{O} 8^{\mathrm{v}}-\mathrm{Mg} 3-\mathrm{O}^{\mathrm{xviii}}$ & $76.80(3)$ \\
\hline $\mathrm{A} 13 \mathrm{~A}^{\mathrm{xvii}}-\mathrm{A} 13 \mathrm{~A}-\mathrm{Cr} 3 \mathrm{~B}^{\mathrm{xx}}$ & 60.0 & $\mathrm{O} 3^{\mathrm{xvii}}-\mathrm{Mg} 3-\mathrm{Al} 3 \mathrm{~A}$ & $82.33(2)$ \\
\hline $\mathrm{A} 13 \mathrm{~A}^{\mathrm{xx}}-\mathrm{A} 13 \mathrm{~A}-\mathrm{Cr} 3 \mathrm{~B}^{\mathrm{xx}}$ & $0.000(14)$ & $\mathrm{O} 3^{\mathrm{ii}}-\mathrm{Mg} 3-\mathrm{Al} 3 \mathrm{~A}$ & $126.66(2)$ \\
\hline $\mathrm{O} 6-\mathrm{A} 13 \mathrm{~A}-\mathrm{Cr} 3 \mathrm{~B}^{\mathrm{iii}}$ & $41.52(2)$ & 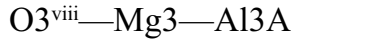 & $126.66(2)$ \\
\hline $\mathrm{O}^{\mathrm{xvii}}-\mathrm{A} 13 \mathrm{~A}-\mathrm{Cr} 3 \mathrm{~B}^{\mathrm{iii}}$ & $137.691(16)$ & $\mathrm{O}^{\mathrm{xvi}}-\mathrm{Mg} 3-\mathrm{A} 13 \mathrm{~A}$ & $78.26(3)$ \\
\hline $\mathrm{O} 5-\mathrm{Al} 3 \mathrm{~A}-\mathrm{Cr} 3 \mathrm{~B}^{\mathrm{iii}}$ & 94.703 (19) & $\mathrm{O} 8 \mathrm{v}-\mathrm{Mg} 3-\mathrm{Al} 3 \mathrm{~A}$ & $38.697(14)$ \\
\hline $\mathrm{O} 8^{\mathrm{v}}-\mathrm{A} 13 \mathrm{~A}-\mathrm{Cr} 3 \mathrm{~B}^{\mathrm{iii}}$ & $42.266(18)$ & 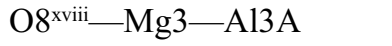 & $38.697(14)$ \\
\hline $\mathrm{O}^{\mathrm{xvii}}-\mathrm{Al} 3 \mathrm{~A}-\mathrm{Cr} 3 \mathrm{~B}^{\mathrm{iii}}$ & $88.730(18)$ & $\mathrm{O} 3^{\mathrm{xvii}}-\mathrm{Mg} 3-\mathrm{A} 13 \mathrm{~A}^{\mathrm{iii}}$ & $126.66(2)$ \\
\hline $\mathrm{O}^{\mathrm{xix}}-\mathrm{A} 13 \mathrm{~A}-\mathrm{Cr} 3 \mathrm{~B}^{\mathrm{iii}}$ & $137.552(19)$ & $\mathrm{O}^{3 i}-\mathrm{Mg} 3-\mathrm{Al} 3 \mathrm{~A}^{\mathrm{iii}}$ & $82.33(2)$ \\
\hline $\mathrm{Cr} 3 \mathrm{~B}^{\mathrm{xvii}}-\mathrm{Al} 3 \mathrm{~A}-\mathrm{Cr} 3 \mathrm{~B}^{\mathrm{iii}}$ & 180.0 & $\mathrm{O} 3^{\text {viii_- }} \mathrm{Mg} 3-\mathrm{A} 13 \mathrm{~A}^{\mathrm{iii}}$ & $126.66(2)$ \\
\hline $\mathrm{Al} 3 \mathrm{~A}^{\mathrm{xvii}}-\mathrm{Al} 3 \mathrm{~A}-\mathrm{Cr} 3 \mathrm{~B}^{\mathrm{iii}}$ & 180.0 & $\mathrm{O} 8^{\mathrm{xvi}}-\mathrm{Mg} 3-\mathrm{Al} 3 \mathrm{~A}^{\mathrm{iii}}$ & 38.697 (14) \\
\hline $\mathrm{A} 13 \mathrm{~A}^{\mathrm{xx}}-\mathrm{Al} 3 \mathrm{~A}-\mathrm{Cr} 3 \mathrm{~B}^{\mathrm{iii}}$ & 120.0 & $\mathrm{O} 8^{\mathrm{v}}-\mathrm{Mg} 3-\mathrm{Al} 3 \mathrm{~A}^{\mathrm{iii}}$ & $38.697(14)$ \\
\hline $\mathrm{Cr} 3 \mathrm{~B}^{\mathrm{xx}}-\mathrm{A} 13 \mathrm{~A}-\mathrm{Cr} 3 \mathrm{~B}^{\mathrm{iii}}$ & 120.0 & $\mathrm{O} 8^{\mathrm{xvii}}-\mathrm{Mg} 3-\mathrm{A} 13 \mathrm{~A}^{\mathrm{iii}}$ & $78.26(3)$ \\
\hline $\mathrm{O} 6-\mathrm{Al} 3 \mathrm{~A}-\mathrm{Al} 3 \mathrm{~A}^{\mathrm{iii}}$ & $41.52(2)$ & $\mathrm{Al} 3 \mathrm{~A}-\mathrm{Mg} 3-\mathrm{A} 13 \mathrm{~A}^{\mathrm{iii}}$ & $55.352(15)$ \\
\hline $\mathrm{O}^{\mathrm{xvii}}-\mathrm{A} 13 \mathrm{~A}-\mathrm{A} 13 \mathrm{~A}^{\mathrm{iii}}$ & $137.691(16)$ & $\mathrm{O}^{\mathrm{xvii}}-\mathrm{Mg} 3-\mathrm{Cr} 3 \mathrm{~B}^{\mathrm{iii}}$ & $126.66(2)$ \\
\hline $\mathrm{O} 5-\mathrm{Al} 3 \mathrm{~A}-\mathrm{Al} 3 \mathrm{~A}^{\mathrm{iii}}$ & 94.703 (19) & $\mathrm{O}^{3 i}-\mathrm{Mg} 3-\mathrm{Cr}^{\mathrm{ii}} \mathrm{B}^{\mathrm{iii}}$ & $82.33(2)$ \\
\hline $\mathrm{O} 8^{\mathrm{v}}-\mathrm{A} 13 \mathrm{~A}-\mathrm{Al} 3 \mathrm{~A}^{\mathrm{iii}}$ & $42.266(18)$ & 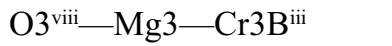 & $126.66(2)$ \\
\hline $\mathrm{O} 8^{\mathrm{xvii}}-\mathrm{A} 13 \mathrm{~A}-\mathrm{A} 13 \mathrm{~A}^{\mathrm{iii}}$ & $88.730(18)$ & $\mathrm{O} 8^{\mathrm{xvi}}-\mathrm{Mg} 3-\mathrm{Cr} 3 \mathrm{~B}^{\mathrm{iii}}$ & $38.697(14)$ \\
\hline $\mathrm{O}^{\mathrm{xix}}-\mathrm{Al} 3 \mathrm{~A}-\mathrm{Al} 3 \mathrm{~A}^{\mathrm{iii}}$ & $137.552(19)$ & $\mathrm{O} 8^{\mathrm{v}}-\mathrm{Mg} 3-\mathrm{Cr} 3 \mathrm{~B}^{\mathrm{iii}}$ & 38.697 (14) \\
\hline $\mathrm{Cr} 3 \mathrm{~B}^{\mathrm{xvii}}-\mathrm{Al} 3 \mathrm{~A}-\mathrm{Al} 3 \mathrm{~A}^{\mathrm{iii}}$ & 180.0 & $\mathrm{O} 8^{\mathrm{xviii}}-\mathrm{Mg} 3-\mathrm{Cr} 3 \mathrm{~B}^{\mathrm{iii}}$ & $78.26(3)$ \\
\hline $\mathrm{Al} 3 \mathrm{~A}^{\mathrm{xvii}}-\mathrm{Al} 3 \mathrm{~A}-\mathrm{Al} 3 \mathrm{~A}^{\mathrm{iii}}$ & 180.0 & $\mathrm{Al} 3 \mathrm{~A}-\mathrm{Mg} 3-\mathrm{Cr} 3 \mathrm{~B}^{\mathrm{iii}}$ & 55.4 \\
\hline $\mathrm{Al} 3 \mathrm{~A}^{\mathrm{xx}}-\mathrm{Al} 3 \mathrm{~A}-\mathrm{Al} 3 \mathrm{~A}^{\mathrm{iii}}$ & 120.0 & $\mathrm{Al} 3 \mathrm{~A}^{\mathrm{iii}}-\mathrm{Mg} 3-\mathrm{Cr} \mathrm{B}^{\mathrm{iii}}$ & $0.000(16)$ \\
\hline $\mathrm{Cr} 3 \mathrm{~B}^{\mathrm{xx}}-\mathrm{Al} 3 \mathrm{~A}-\mathrm{Al} 3 \mathrm{~A}^{\mathrm{iii}}$ & 120.0 & $\mathrm{O}^{\text {xvii }}-\mathrm{Mg} 3-\mathrm{Al} 3 \mathrm{~A}^{\mathrm{iv}}$ & $126.66(2)$ \\
\hline $\mathrm{Cr} 3 \mathrm{~B}^{\mathrm{iii}}-\mathrm{A} 13 \mathrm{~A}-\mathrm{Al} 3 \mathrm{~A}^{\mathrm{iii}}$ & 0.0 & $\mathrm{O}^{3}-\mathrm{ii}-\mathrm{Mg} 3-\mathrm{Al}^{3} \mathrm{~A}^{\mathrm{iv}}$ & $126.66(2)$ \\
\hline $\mathrm{O} 1^{\mathrm{xxi}}-\mathrm{A} 14 \mathrm{~A}-\mathrm{O} 1$ & 180.0 & $\mathrm{O} 3^{\text {viii }}-\mathrm{Mg} 3-\mathrm{Al} 3 \mathrm{~A}^{\mathrm{iv}}$ & $82.33(2)$ \\
\hline $\mathrm{O} 1^{\mathrm{xxi}}-\mathrm{A} 14 \mathrm{~A}-\mathrm{O} 4^{\mathrm{xxii}}$ & $96.18(3)$ & $\mathrm{O}^{\mathrm{xvi}}-\mathrm{Mg} 3-\mathrm{Al} 3 \mathrm{~A}^{\mathrm{iv}}$ & $38.697(14)$ \\
\hline $\mathrm{O} 1-\mathrm{A} 14 \mathrm{~A}-\mathrm{O} 4^{\mathrm{xxii}}$ & $83.82(3)$ & $\mathrm{O} 8 \mathrm{v}-\mathrm{Mg} 3-\mathrm{A} 13 \mathrm{~A}^{\mathrm{iv}}$ & $78.26(3)$ \\
\hline $\mathrm{O} 1^{\mathrm{xxi}}-\mathrm{Al} 4 \mathrm{~A}-\mathrm{O} 4^{\mathrm{x}}$ & $83.82(3)$ & $\mathrm{O} 8^{\mathrm{xvii}}-\mathrm{Mg} 3-\mathrm{Al} 3 \mathrm{~A}^{\mathrm{iv}}$ & $38.697(14)$ \\
\hline $\mathrm{O} 1-\mathrm{Al} 4 \mathrm{~A}-\mathrm{O} 4^{\mathrm{x}}$ & $96.18(3)$ & $\mathrm{Al} 3 \mathrm{~A}-\mathrm{Mg} 3-\mathrm{Al} 3 \mathrm{~A}^{\mathrm{iv}}$ & $55.352(15)$ \\
\hline
\end{tabular}




\begin{tabular}{|c|c|c|c|}
\hline $\mathrm{O} 4^{\mathrm{xxii}}-\mathrm{A} 14 \mathrm{~A}-\mathrm{O} 4^{\mathrm{x}}$ & $180.00(3)$ & $\mathrm{A} 13 \mathrm{~A}^{\mathrm{iii}}-\mathrm{Mg} 3-\mathrm{Al} 3 \mathrm{~A}^{\mathrm{iv}}$ & $55.351(15)$ \\
\hline $\mathrm{O} 1^{\mathrm{xxi}}-\mathrm{A} 14 \mathrm{~A}-\mathrm{O} 4^{\mathrm{xxiii}}$ & $96.18(3)$ & $\mathrm{Cr} 3 \mathrm{~B}^{\mathrm{iii}}-\mathrm{Mg} 3-\mathrm{Al} 3 \mathrm{~A}^{\mathrm{iv}}$ & 55.4 \\
\hline $\mathrm{O} 1-\mathrm{A} 14 \mathrm{~A}-\mathrm{O} 4^{\mathrm{x} x i i \mathrm{i}}$ & $83.82(3)$ & $\mathrm{O} 3^{\mathrm{xvii}}-\mathrm{Mg} 3-\mathrm{Cr} 3 \mathrm{~B}^{\mathrm{iv}}$ & $126.66(2)$ \\
\hline $\mathrm{O} 4^{\mathrm{xxii}}-\mathrm{A} 14 \mathrm{~A}-\mathrm{O} 4^{\mathrm{xxiii}}$ & $83.33(4)$ & $\mathrm{O}^{\mathrm{ii}}-\mathrm{Mg} 3-\mathrm{Cr} 3 \mathrm{~B}^{\mathrm{iv}}$ & $126.66(2)$ \\
\hline $\mathrm{O} 4^{\mathrm{x}}-\mathrm{A} 14 \mathrm{~A}-\mathrm{O} 4^{\mathrm{xxiii}}$ & $96.67(4)$ & $\mathrm{O}^{\text {viii }}-\mathrm{Mg} 3-\mathrm{Cr} 3 \mathrm{~B}^{\text {iv }}$ & $82.33(2)$ \\
\hline $\mathrm{O} 1^{\mathrm{xxi}}-\mathrm{A} 14 \mathrm{~A}-\mathrm{O} 4^{\mathrm{viii}}$ & $83.82(3)$ & $\mathrm{O} 8^{\mathrm{xvi}}-\mathrm{Mg} 3-\mathrm{Cr} 3 \mathrm{~B}^{\mathrm{iv}}$ & $38.697(14)$ \\
\hline $\mathrm{O} 1-\mathrm{A} 14 \mathrm{~A}-\mathrm{O} 4^{\mathrm{viii}}$ & $96.18(3)$ & $\mathrm{O} 8 \mathrm{v}-\mathrm{Mg} 3-\mathrm{Cr} 3 \mathrm{~B}^{\mathrm{iv}}$ & $78.26(3)$ \\
\hline $\mathrm{O} 4^{\mathrm{xxii}}-\mathrm{A} 14 \mathrm{~A}-\mathrm{O} 4^{\text {viii }}$ & $96.67(4)$ & $\mathrm{O}^{\mathrm{xvii}}-\mathrm{Mg} 3-\mathrm{Cr} 3 \mathrm{~B}^{\mathrm{iv}}$ & $38.697(14)$ \\
\hline $\mathrm{O} 4^{\mathrm{x}}-\mathrm{A} 14 \mathrm{~A}-\mathrm{O} 4^{\mathrm{viii}}$ & $83.33(4)$ & $\mathrm{A} 13 \mathrm{~A}-\mathrm{Mg} 3-\mathrm{Cr} 3 \mathrm{~B}^{\mathrm{iv}}$ & 55.4 \\
\hline $\mathrm{O} 4^{\mathrm{xxiii}}-\mathrm{A} 14 \mathrm{~A}-\mathrm{O} 4^{\mathrm{viii}}$ & 180.0 & $\mathrm{~A} 13 \mathrm{~A}^{\mathrm{iii}}-\mathrm{Mg} 3-\mathrm{Cr} 3 \mathrm{~B}^{\mathrm{iv}}$ & $55.351(15)$ \\
\hline $\mathrm{O}^{\mathrm{xxi}}-\mathrm{A} 14 \mathrm{~A}-\mathrm{A} 14 \mathrm{~A}^{\mathrm{xiv}}$ & $42.07(2)$ & $\mathrm{Cr} 3 \mathrm{~B}^{\mathrm{iii}}-\mathrm{Mg} 3-\mathrm{Cr} 3 \mathrm{~B}^{\mathrm{iv}}$ & $55.351(15)$ \\
\hline $\mathrm{O} 1-\mathrm{A} 14 \mathrm{~A}-\mathrm{A} 14 \mathrm{~A}^{\mathrm{xiv}}$ & $137.93(2)$ & $\mathrm{Al} 3 \mathrm{~A}^{\mathrm{iv}}-\mathrm{Mg} 3-\mathrm{Cr} 3 \mathrm{~B}^{\mathrm{iv}}$ & $0.000(6)$ \\
\hline $\mathrm{O} 4^{\mathrm{xxii}}-\mathrm{A} 14 \mathrm{~A}-\mathrm{A} 14 \mathrm{~A}^{\mathrm{xiv}}$ & $94.432(19)$ & $\mathrm{Cr} 4 \mathrm{~B}^{\mathrm{xv}}-\mathrm{O} 1-\mathrm{Al} 4 \mathrm{~A}^{\mathrm{xv}}$ & 0.0 \\
\hline $\mathrm{O} 4^{\mathrm{x}}-\mathrm{A} 14 \mathrm{~A}-\mathrm{A} 14 \mathrm{~A}^{\mathrm{xiv}}$ & $85.568(19)$ & $\mathrm{Cr} 4 \mathrm{~B}^{\mathrm{xv}}-\mathrm{O} 1-\mathrm{A} 14 \mathrm{~A}$ & 95.9 \\
\hline $\mathrm{O} 4^{\mathrm{xxii}}-\mathrm{A} 14 \mathrm{~A}-\mathrm{A} 14 \mathrm{~A}^{\mathrm{xiv}}$ & $137.904(15)$ & $\mathrm{Al} 4 \mathrm{~A}^{\mathrm{xv}}-\mathrm{O} 1-\mathrm{Al} 4 \mathrm{~A}$ & $95.86(4)$ \\
\hline $\mathrm{O} 4^{\text {viii }}-\mathrm{Al} 4 \mathrm{~A}-\mathrm{Al} 4 \mathrm{~A}^{\mathrm{xiv}}$ & $42.096(15)$ & $\mathrm{Cr} 4 \mathrm{~B}^{\mathrm{xv}}-\mathrm{O} 1-\mathrm{Cr} 4 \mathrm{~B}^{\mathrm{vi}}$ & $95.86(4)$ \\
\hline $\mathrm{O} 1^{\mathrm{xxi}}-\mathrm{A} 14 \mathrm{~A}-\mathrm{A} 14 \mathrm{~A}^{\mathrm{xv}}$ & $137.93(2)$ & $\mathrm{A} 14 \mathrm{~A}^{\mathrm{xv}}-\mathrm{O} 1-\mathrm{Cr} 4 \mathrm{~B}^{\mathrm{vi}}$ & $95.86(4)$ \\
\hline $\mathrm{O} 1-\mathrm{Al} 4 \mathrm{~A}-\mathrm{Al} 4 \mathrm{~A}^{\mathrm{xv}}$ & $42.07(2)$ & $\mathrm{A} 14 \mathrm{~A}-\mathrm{O} 1-\mathrm{Cr} 4 \mathrm{~B}^{\mathrm{vi}}$ & 95.9 \\
\hline $\mathrm{O} 4^{\mathrm{xxii}}-\mathrm{A} 14 \mathrm{~A}-\mathrm{A} 14 \mathrm{~A}^{\mathrm{xv}}$ & $85.568(19)$ & $\mathrm{Cr} 4 \mathrm{~B}^{\mathrm{xv}}-\mathrm{O} 1-\mathrm{Al}^{4} \mathrm{~A}^{\mathrm{vi}}$ & 95.9 \\
\hline $\mathrm{O} 4^{\mathrm{x}}-\mathrm{A} 14 \mathrm{~A}-\mathrm{A} 14 \mathrm{~A}^{\mathrm{xv}}$ & $94.432(19)$ & $\mathrm{A} 14 \mathrm{~A}^{\mathrm{xv}}-\mathrm{O} 1-\mathrm{Al} 4 \mathrm{~A}^{\mathrm{vi}}$ & $95.86(4)$ \\
\hline $\mathrm{O} 4^{\mathrm{xxiii}}-\mathrm{Al} 4 \mathrm{~A}-\mathrm{Al} 4 \mathrm{~A}^{\mathrm{xv}}$ & $42.096(15)$ & $\mathrm{Al} 4 \mathrm{~A}-\mathrm{O} 1-\mathrm{Al}^{\mathrm{vi}}$ & $95.86(4)$ \\
\hline $\mathrm{O} 4^{\mathrm{viii}}-\mathrm{A} 14 \mathrm{~A}-\mathrm{Al} 4 \mathrm{~A}^{\mathrm{xv}}$ & $137.904(15)$ & $\mathrm{Cr} 4 \mathrm{~B}^{\mathrm{vi}}-\mathrm{O} 1-\mathrm{Al} 4 \mathrm{~A}^{\mathrm{vi}}$ & 0.0 \\
\hline $\mathrm{A} 14 \mathrm{~A}^{\mathrm{xiv}}-\mathrm{A} 14 \mathrm{~A}-\mathrm{A} 14 \mathrm{~A}^{\mathrm{xv}}$ & 180.0 & $\mathrm{Cr} 4 \mathrm{~B}^{\mathrm{xv}}-\mathrm{O} 1-\mathrm{Mg} 2 \mathrm{~A}$ & $121.00(3)$ \\
\hline $\mathrm{O} 1^{\mathrm{xxi}}-\mathrm{A} 14 \mathrm{~A}-\mathrm{Cr} 4 \mathrm{~B}^{\mathrm{xiv}}$ & $42.07(2)$ & $\mathrm{A} 14 \mathrm{~A}^{\mathrm{xv}}-\mathrm{O} 1-\mathrm{Mg} 2 \mathrm{~A}$ & $121.00(3)$ \\
\hline $\mathrm{O} 1-\mathrm{A} 14 \mathrm{~A}-\mathrm{Cr} 4 \mathrm{~B}^{\mathrm{xiv}}$ & $137.93(2)$ & $\mathrm{A} 14 \mathrm{~A}-\mathrm{O} 1-\mathrm{Mg} 2 \mathrm{~A}$ & $121.00(3)$ \\
\hline $\mathrm{O} 4^{\mathrm{xxii}}-\mathrm{A} 14 \mathrm{~A}-\mathrm{Cr} 4 \mathrm{~B}^{\mathrm{xiv}}$ & 94.432 (19) & $\mathrm{Cr} 4 \mathrm{~B}^{\mathrm{vi}}-\mathrm{O} 1-\mathrm{Mg} 2 \mathrm{~A}$ & $121.00(3)$ \\
\hline $\mathrm{O} 4^{\mathrm{x}}-\mathrm{A} 14 \mathrm{~A}-\mathrm{Cr} 4 \mathrm{~B}^{\mathrm{xiv}}$ & $85.568(19)$ & $\mathrm{A} 14 \mathrm{~A}^{\mathrm{vi}}-\mathrm{O} 1-\mathrm{Mg} 2 \mathrm{~A}$ & $121.00(3)$ \\
\hline $\mathrm{O} 4^{\text {xxii }}-\mathrm{Al} 4 \mathrm{~A}-\mathrm{Cr} 4 \mathrm{~B}^{\mathrm{xiv}}$ & $137.904(15)$ & $\mathrm{A} 12-\mathrm{O} 2-\mathrm{A} 11$ & $123.53(3)$ \\
\hline $\mathrm{O} 4^{\mathrm{viii}}-\mathrm{Al} 4 \mathrm{~A}-\mathrm{Cr} 4 \mathrm{~B}^{\mathrm{xiv}}$ & $42.096(15)$ & $\mathrm{A} 12-\mathrm{O} 2-\mathrm{Al} 1^{\mathrm{xv}}$ & $123.53(3)$ \\
\hline $\mathrm{A} 14 \mathrm{~A}^{\mathrm{xiv}}-\mathrm{A} 14 \mathrm{~A}-\mathrm{Cr} 4 \mathrm{~B}^{\mathrm{xiv}}$ & 0.0 & $\mathrm{All}-\mathrm{O} 2-\mathrm{Al1}{ }^{\mathrm{xv}}$ & $92.42(4)$ \\
\hline $\mathrm{Al} 4 \mathrm{~A}^{\mathrm{xv}}-\mathrm{Al} 4 \mathrm{~A}-\mathrm{Cr} 4 \mathrm{~B}^{\mathrm{xiv}}$ & 180.0 & $\mathrm{~A} 12-\mathrm{O} 2-\mathrm{A} 11^{\mathrm{vi}}$ & $123.53(3)$ \\
\hline $\mathrm{O} 1^{\mathrm{xxi}}-\mathrm{A} 14 \mathrm{~A}-\mathrm{Cr} 4 \mathrm{~B}^{\mathrm{xv}}$ & $137.93(2)$ & $\mathrm{A} 11-\mathrm{O} 2-\mathrm{A} 11^{\mathrm{vi}}$ & $92.42(4)$ \\
\hline $\mathrm{O} 1-\mathrm{A} 14 \mathrm{~A}-\mathrm{Cr} 4 \mathrm{~B}^{\mathrm{xv}}$ & $42.07(2)$ & $\mathrm{A} 11^{\mathrm{xv}}-\mathrm{O} 2-\mathrm{A} 11^{\mathrm{vi}}$ & $92.42(4)$ \\
\hline $\mathrm{O} 4^{\mathrm{xxii}}-\mathrm{A} 14 \mathrm{~A}-\mathrm{Cr} 4 \mathrm{~B}^{\mathrm{xv}}$ & $85.568(19)$ & $\mathrm{Be} 1^{\mathrm{xix}}-\mathrm{O} 3-\mathrm{A} 11^{\mathrm{xviii}}$ & $89.25(5)$ \\
\hline $\mathrm{O} 4^{\mathrm{x}}-\mathrm{A} 14 \mathrm{~A}-\mathrm{Cr} 4 \mathrm{~B}^{\mathrm{xv}}$ & $94.432(19)$ & $\mathrm{Be} 1^{\mathrm{xix}}-\mathrm{O} 3-\mathrm{A} 11^{\mathrm{xxv}}$ & $89.25(5)$ \\
\hline $\mathrm{O} 4^{\mathrm{xxiii}}-\mathrm{A} 14 \mathrm{~A}-\mathrm{Cr} 4 \mathrm{~B}^{\mathrm{xv}}$ & $42.096(15)$ & $\mathrm{A} 11^{\mathrm{xviii}}-\mathrm{O} 3-\mathrm{A} 11^{\mathrm{xxv}}$ & $98.09(3)$ \\
\hline $\mathrm{O} 4^{\mathrm{viii}}-\mathrm{A} 14 \mathrm{~A}-\mathrm{Cr} 4 \mathrm{~B}^{\mathrm{xv}}$ & $137.904(15)$ & $\mathrm{Be}^{\mathrm{xix}}-\mathrm{O} 3-\mathrm{Mg} 3^{\mathrm{xxv}}$ & $125.79(8)$ \\
\hline $\mathrm{A} 14 \mathrm{~A}^{\mathrm{xiv}}-\mathrm{A} 14 \mathrm{~A}-\mathrm{Cr} 4 \mathrm{~B}^{\mathrm{xv}}$ & 180.0 & $\mathrm{~A} 11^{\mathrm{xviii}}-\mathrm{O} 3-\mathrm{Mg} 3^{\mathrm{xxv}}$ & $122.63(2)$ \\
\hline $\mathrm{Al} 4 \mathrm{~A}^{\mathrm{xv}}-\mathrm{Al} 4 \mathrm{~A}-\mathrm{Cr} 4 \mathrm{~B}^{\mathrm{xv}}$ & 0.0 & $\mathrm{~A} 11^{\mathrm{xxv}}-\mathrm{O} 3-\mathrm{Mg}^{\mathrm{xxv}}$ & $122.63(2)$ \\
\hline $\mathrm{Cr} 4 \mathrm{~B}^{\mathrm{xiv}}-\mathrm{A} 14 \mathrm{~A}-\mathrm{Cr} 4 \mathrm{~B}^{\mathrm{xv}}$ & 180.0 & $\mathrm{Cr} 4 \mathrm{~B}^{\mathrm{xxv}}-\mathrm{O} 4-\mathrm{A} 14 \mathrm{~A}^{\mathrm{xxv}}$ & 0.0 \\
\hline $\mathrm{O} 1^{\mathrm{xxi}}-\mathrm{A} 14 \mathrm{~A}-\mathrm{A} 14 \mathrm{~A}^{\mathrm{vi}}$ & $137.93(2)$ & $\mathrm{Cr} 4 \mathrm{~B}^{\mathrm{xxv}}-\mathrm{O} 4-\mathrm{Cr} 4 \mathrm{~B}^{\mathrm{xviii}}$ & $95.81(3)$ \\
\hline $\mathrm{O} 1-\mathrm{A} 14 \mathrm{~A}-\mathrm{Al} 4 \mathrm{~A}^{\mathrm{vi}}$ & $42.07(2)$ & $\mathrm{A} 14 \mathrm{~A}^{\mathrm{xxv}}-\mathrm{O} 4-\mathrm{Cr} 4 \mathrm{~B}^{\mathrm{xviii}}$ & $95.81(3)$ \\
\hline $\mathrm{O} 4^{\mathrm{xxii}}-\mathrm{Al} 4 \mathrm{~A}-\mathrm{Al} 4 \mathrm{~A}^{\mathrm{vi}}$ & $42.096(15)$ & $\mathrm{Cr} 4 \mathrm{~B}^{\mathrm{xxv}}-\mathrm{O} 4-\mathrm{A} 14 \mathrm{~A}^{\mathrm{xviii}}$ & 95.8 \\
\hline $\mathrm{O} 4^{\mathrm{x}}-\mathrm{A} 14 \mathrm{~A}-\mathrm{Al} 4 \mathrm{~A}^{\mathrm{vi}}$ & $137.904(15)$ & $\mathrm{A} 14 \mathrm{~A}^{\mathrm{xxv}}-\mathrm{O} 4-\mathrm{A} 14 \mathrm{~A}^{\mathrm{xviii}}$ & $95.81(3)$ \\
\hline $\mathrm{O} 4^{\mathrm{xxiii}}-\mathrm{Al} 4 \mathrm{~A}-\mathrm{Al} 4 \mathrm{~A}^{\mathrm{vi}}$ & $85.569(18)$ & $\mathrm{Cr} 4 \mathrm{~B}^{\mathrm{xviii}}-\mathrm{O} 4-\mathrm{Al} 4 \mathrm{~A}^{\mathrm{xviii}}$ & 0.0 \\
\hline $\mathrm{O} 4^{\mathrm{viii}}-\mathrm{A} 14 \mathrm{~A}-\mathrm{Al} 4 \mathrm{~A}^{\mathrm{vi}}$ & $94.431(18)$ & $\mathrm{Cr} 4 \mathrm{~B}^{\mathrm{xxy}}-\mathrm{O} 4-\mathrm{Al}^{\mathrm{xix}}$ & $96.34(3)$ \\
\hline $\mathrm{Al} 4 \mathrm{~A}^{\mathrm{xiv}}-\mathrm{Al} 4 \mathrm{~A}-\mathrm{Al} 4 \mathrm{~A}^{\mathrm{vi}}$ & 120.0 & $\mathrm{Al} 4 \mathrm{~A}^{\mathrm{xxy}}-\mathrm{O} 4-\mathrm{Al} 5^{\mathrm{xix}}$ & $96.34(3)$ \\
\hline $\mathrm{Al} 4 \mathrm{~A}^{\mathrm{xv}}-\mathrm{A} 14 \mathrm{~A}-\mathrm{Al} 4 \mathrm{~A}^{\mathrm{vi}}$ & 60.0 & $\mathrm{Cr} 4 \mathrm{~B}^{\mathrm{xviii}}-\mathrm{O} 4-\mathrm{Al}^{\mathrm{xix}}$ & $96.34(3)$ \\
\hline
\end{tabular}


Cr4B ${ }^{\text {xiv }}-\mathrm{A} 14 \mathrm{~A}-\mathrm{A} 14 \mathrm{~A}^{\mathrm{vi}}$

$\mathrm{Cr}_{4} \mathrm{~B}^{\mathrm{xv}}-\mathrm{Al} 4 \mathrm{~A}-\mathrm{Al}^{\mathrm{A}} \mathrm{A}^{\mathrm{vi}}$

$\mathrm{O} 1^{\mathrm{xxi}}-\mathrm{A} 14 \mathrm{~A}-\mathrm{Cr} 4 \mathrm{~B}^{\mathrm{vi}}$

$\mathrm{O} 1-\mathrm{A} 14 \mathrm{~A}-\mathrm{Cr} 4 \mathrm{~B}^{\mathrm{vi}}$

$\mathrm{O} 4^{\mathrm{xxi}}-\mathrm{Al} 4 \mathrm{~A}-\mathrm{Cr} 4 \mathrm{~B}^{\mathrm{vi}}$

$\mathrm{O} 4^{\mathrm{x}}-\mathrm{A} 14 \mathrm{~A}-\mathrm{Cr}^{4} \mathrm{~B}^{\mathrm{vi}}$

$\mathrm{O} 4^{\mathrm{xxiii}}-\mathrm{A} 14 \mathrm{~A}-\mathrm{Cr} 4 \mathrm{~B}^{\mathrm{vi}}$

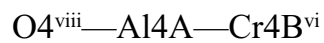

$\mathrm{Al}^{\mathrm{A}} \mathrm{A}^{\mathrm{xiv}}-\mathrm{A} 14 \mathrm{~A}-\mathrm{Cr} 4 \mathrm{~B}^{\mathrm{vi}}$

$\mathrm{A} 14 \mathrm{~A}^{\mathrm{xv}}-\mathrm{A} 14 \mathrm{~A}-\mathrm{Cr} 4 \mathrm{~B}^{\mathrm{vi}}$

$\mathrm{Cr} 4 \mathrm{~B}^{\mathrm{xiv}}-\mathrm{A} 14 \mathrm{~A}-\mathrm{Cr} 4 \mathrm{~B}^{\mathrm{vi}}$

$\mathrm{Cr} 4 \mathrm{~B}^{\mathrm{xv}}-\mathrm{A} 14 \mathrm{~A}-\mathrm{Cr} 4 \mathrm{~B}^{\mathrm{vi}}$

$\mathrm{Al} 4 \mathrm{~A}^{\mathrm{vi}}-\mathrm{A} 14 \mathrm{~A}-\mathrm{Cr} 4 \mathrm{~B}^{\mathrm{vi}}$

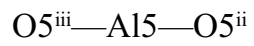

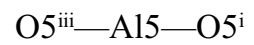

$\mathrm{O} 5^{\mathrm{ii}}-\mathrm{A} 15-\mathrm{O}^{\mathrm{i}}$

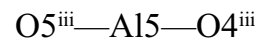

$\mathrm{O} 5^{\mathrm{ii}}-\mathrm{A} 15-\mathrm{O} 4^{\mathrm{iii}}$

$\mathrm{O} 5^{\mathrm{i}}-\mathrm{A} 15-\mathrm{O} 4^{\mathrm{iii}}$

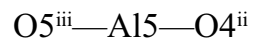

$\mathrm{O} 5^{\mathrm{ii}}-\mathrm{A} 15-\mathrm{O} 4^{\mathrm{ii}}$

$\mathrm{O} 5^{\mathrm{i}}-\mathrm{A} 15-\mathrm{O} 4^{\mathrm{ii}}$

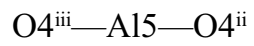

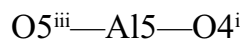

$\mathrm{O} 5^{\mathrm{ii}}-\mathrm{A} 15-\mathrm{O} 4^{\mathrm{i}}$

$\mathrm{O} 5^{\mathrm{i}}-\mathrm{A} 15-\mathrm{O} 4^{\mathrm{i}}$

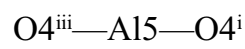

$\mathrm{O} 4^{\mathrm{ii}}-\mathrm{A} 15-\mathrm{O} 4^{\mathrm{i}}$

$\mathrm{O}^{\mathrm{iii}}-\mathrm{A} 15-\mathrm{Cr} 4 \mathrm{~B}^{\mathrm{v}}$

$\mathrm{O}^{\mathrm{ii}}-\mathrm{A} 15-\mathrm{Cr} 4 \mathrm{~B}^{\mathrm{v}}$

$\mathrm{O}^{\mathrm{i}}-\mathrm{A} 15-\mathrm{Cr}^{4} \mathrm{~B}^{\mathrm{v}}$

$\mathrm{O} 4{ }^{\mathrm{iii}}-\mathrm{A} 15-\mathrm{Cr}_{4} \mathrm{~B}^{\mathrm{v}}$

$\mathrm{O} 4{ }^{\mathrm{ii}}-\mathrm{A} 15-\mathrm{Cr} 4 \mathrm{~B}^{\mathrm{v}}$

$\mathrm{O} 4-\mathrm{A} 15-\mathrm{Cr}^{\mathrm{i}} \mathrm{B}^{\mathrm{v}}$

O5 ${ }^{\mathrm{iii}}-\mathrm{A} 15-\mathrm{A} 14 \mathrm{~A}^{\mathrm{v}}$

$\mathrm{O}^{\mathrm{ii}}-\mathrm{A} 15-\mathrm{Al}^{\mathrm{v}} \mathrm{A}$

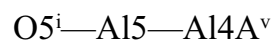

$\mathrm{O} 4{ }^{\mathrm{iii}}-\mathrm{A} 15-\mathrm{A} 14 \mathrm{~A}^{\mathrm{v}}$

$\mathrm{O} 4{ }^{\mathrm{ii}}-\mathrm{A} 15-\mathrm{A} 14 \mathrm{~A}^{\mathrm{v}}$

$\mathrm{O} 4 \mathrm{i}-\mathrm{A} 15-\mathrm{A} 14 \mathrm{~A}^{\mathrm{v}}$

$\mathrm{Cr} 4 \mathrm{~B}^{\mathrm{v}}-\mathrm{Al} 5-\mathrm{Al}_{4} \mathrm{~A}^{\mathrm{v}}$

$\mathrm{O}^{\mathrm{iii}}-\mathrm{A} 15-\mathrm{Cr} 4 \mathrm{~B}^{\mathrm{iv}}$

$\mathrm{O} 5$ ii $-\mathrm{A} 15-\mathrm{Cr} 4 \mathrm{~B}^{\mathrm{iv}}$

$\mathrm{O} 5^{\mathrm{i}}-\mathrm{A} 15-\mathrm{Cr} 4 \mathrm{~B}^{\mathrm{iv}}$

$\mathrm{O} 4{ }^{\mathrm{iii}}-\mathrm{A} 15-\mathrm{Cr} 4 \mathrm{~B}^{\mathrm{iv}}$

$\mathrm{O} 4{ }^{\mathrm{ii}}-\mathrm{A} 15-\mathrm{Cr} 4 \mathrm{~B}^{\mathrm{iv}}$

$\mathrm{O} 4-\mathrm{A} 15-\mathrm{Cr} 4 \mathrm{~B}^{\mathrm{iv}}$

$\mathrm{Cr} 4 \mathrm{~B}^{\mathrm{v}}-\mathrm{A} 15-\mathrm{Cr} 4 \mathrm{~B}^{\mathrm{iv}}$
120.0

60.0

137.93 (2)

42.07 (2)

42.096 (15)

$137.904(15)$

85.569 (18)

94.431 (18)

120.0

60.0

120.0

60.0

0.0

83.03 (3)

83.03 (3)

83.02 (3)

96.83 (2)

$179.81(3)$

96.83 (2)

96.83 (2)

96.83 (2)

$179.81(3)$

83.31 (3)

$179.81(3)$

96.83 (2)

96.83 (2)

83.31 (3)

83.31 (3)

$138.301(15)$

138.300 (15)

94.97 (2)

41.825 (15)

85.22 (2)

41.826 (15)

$138.301(15)$

138.300 (15)

94.97 (2)

41.825 (15)

85.22 (2)

41.826 (15)

0.0

138.301 (15)

94.97 (2)

138.300 (15)

85.22 (2)

41.825 (15)

41.826 (15)

59.719 (10)
A14A $\mathrm{A}^{\mathrm{xviii}}-\mathrm{O} 4-\mathrm{A} 15^{\mathrm{xix}}$

$\mathrm{Cr} 4 \mathrm{~B}^{\mathrm{xxv}}-\mathrm{O} 4-\mathrm{Cr} \mathrm{B}^{\mathrm{xxv}}$

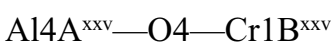

$\mathrm{Cr} 4 \mathrm{~B}^{\mathrm{xviii}}-\mathrm{O} 4-\mathrm{Cr} \mathrm{B}^{\mathrm{xxv}}$

A14A ${ }^{x v i i i}-\mathrm{O} 4-\mathrm{Cr}_{1} \mathrm{~B}^{\mathrm{xxv}}$

$\mathrm{A}^{2} 5^{\mathrm{xix}}-\mathrm{O} 4-\mathrm{Cr}_{1} \mathrm{~B}^{\mathrm{xxv}}$

$\mathrm{Cr} 4 \mathrm{~B}^{\mathrm{xxy}}-\mathrm{O} 4-\mathrm{Mg} 1 \mathrm{~A}^{\mathrm{xxv}}$

$\mathrm{A} 14 \mathrm{~A}^{\mathrm{xxv}}-\mathrm{O} 4-\mathrm{Mg} 1 \mathrm{~A}^{\mathrm{xxv}}$

Cr4B ${ }^{x v i i}-O 4-M g 1 A^{x x v}$

Al4A ${ }^{\text {xviii }-O 4-M g 1 A ~}{ }^{\mathrm{xxv}}$

$\mathrm{A} 15^{\mathrm{xix}}-\mathrm{O} 4-\mathrm{Mg} 1 \mathrm{~A}^{\mathrm{xxv}}$

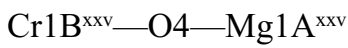

$\mathrm{A} 15^{\mathrm{xix}}-\mathrm{O} 5-\mathrm{Cr} 3 \mathrm{~B}^{\mathrm{xx}}$

$\mathrm{A} 15^{\mathrm{xix}}-\mathrm{O} 5-\mathrm{A} 13 \mathrm{~A}^{\mathrm{xx}}$

$\mathrm{Cr} 3 \mathrm{~B}^{\mathrm{xx}}-\mathrm{O} 5-\mathrm{A} 13 \mathrm{~A}^{\mathrm{xx}}$

$\mathrm{A} 15^{\mathrm{xix}}-\mathrm{O} 5-\mathrm{A} 13 \mathrm{~A}$

$\mathrm{Cr} 3 \mathrm{~B}^{\mathrm{xx}}-\mathrm{O} 5-\mathrm{A} 13 \mathrm{~A}$

$\mathrm{A} 13 \mathrm{~A}^{\mathrm{xx}}-\mathrm{O} 5-\mathrm{A} 13 \mathrm{~A}$

$\mathrm{A} 15^{\mathrm{xix}}-\mathrm{O} 5-\mathrm{Cr} 2 \mathrm{~B}^{\mathrm{xxv}}$

$\mathrm{Cr} 3 \mathrm{~B}^{\mathrm{xx}}-\mathrm{O} 5-\mathrm{Cr} 2 \mathrm{~B}^{\mathrm{xxv}}$

$\mathrm{A} 13 \mathrm{~A}^{\mathrm{xx}}-\mathrm{O} 5-\mathrm{Cr} 2 \mathrm{~B}^{\mathrm{xxv}}$

$\mathrm{A} 13 \mathrm{~A}-\mathrm{O} 5-\mathrm{Cr} 2 \mathrm{~B}^{\mathrm{xxv}}$

$\mathrm{A} 15^{\mathrm{xix}}-\mathrm{O} 5-\mathrm{Mg} 2 \mathrm{~A}^{\mathrm{xxv}}$

$\mathrm{Cr} 3 \mathrm{~B}^{\mathrm{xx}}-\mathrm{O} 5-\mathrm{Mg} 2 \mathrm{~A}^{\mathrm{xxv}}$

$\mathrm{A} 13 \mathrm{~A}^{\mathrm{xx}}-\mathrm{O} 5-\mathrm{Mg} 2 \mathrm{~A}^{\mathrm{xxv}}$

$\mathrm{A} 13 \mathrm{~A}-\mathrm{O} 5-\mathrm{Mg} 2 \mathrm{~A}^{\mathrm{xxv}}$

$\mathrm{Cr} 2 \mathrm{~B}^{\mathrm{xxv}}-\mathrm{O} 5-\mathrm{Mg} 2 \mathrm{~A}^{\mathrm{xxv}}$

$\mathrm{Cr} 3 \mathrm{~B}^{\mathrm{iii}}-\mathrm{O} 6-\mathrm{Al} 3 \mathrm{~A}^{\mathrm{iii}}$

$\mathrm{Cr} 3 \mathrm{~B}^{\mathrm{iii}}-\mathrm{O} 6-\mathrm{Cr} 3 \mathrm{~B}^{\mathrm{iv}}$

$\mathrm{A} 13 \mathrm{~A}^{\mathrm{iii}}-\mathrm{O} 6-\mathrm{Cr} 3 \mathrm{~B}^{\mathrm{iv}}$

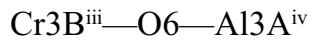

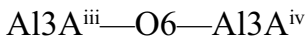

$\mathrm{Cr} 3 \mathrm{~B}^{\mathrm{iv}}-\mathrm{O} 6-\mathrm{Al} 3 \mathrm{~A}^{\mathrm{iv}}$

$\mathrm{Cr} 3 \mathrm{~B}^{\mathrm{iii}}-\mathrm{O} 6-\mathrm{A} 13 \mathrm{~A}$

$\mathrm{Al3} \mathrm{A}^{\mathrm{iii}}-\mathrm{O} 6-\mathrm{A} 13 \mathrm{~A}$

$\mathrm{Cr} 3 \mathrm{~B}^{\mathrm{iv}}-\mathrm{O} 6-\mathrm{A} 13 \mathrm{~A}$

$\mathrm{A} 13 \mathrm{~A}^{\mathrm{iv}}-\mathrm{O} 6-\mathrm{Al} 3 \mathrm{~A}$

$\mathrm{Cr} 3 \mathrm{~B}^{\mathrm{iii}}-\mathrm{O} 6-\mathrm{Mg} 1 \mathrm{~A}$

$\mathrm{Al3A}$ iii_O6-Mg1A

$\mathrm{Cr}_{3} \mathrm{~B}^{\mathrm{iv}}-\mathrm{O} 6-\mathrm{Mg} 1 \mathrm{~A}$

$\mathrm{Al}_{3} \mathrm{~A}^{\mathrm{iv}}-\mathrm{O} 6-\mathrm{Mg} 1 \mathrm{~A}$

$\mathrm{Al3A}-\mathrm{O} 6-\mathrm{Mg} 1 \mathrm{~A}$

$\mathrm{Be} 1-\mathrm{O} 7-\mathrm{Cr}^{3} \mathrm{~B}^{\mathrm{ii}}$

$\mathrm{Be} 1-\mathrm{O} 7-\mathrm{A} 13 \mathrm{~A}^{\mathrm{ii}}$

$\mathrm{Cr} 3 \mathrm{~B}^{\mathrm{ii}}-\mathrm{O} 7-\mathrm{A} 13 \mathrm{~A}^{\mathrm{ii}}$

$\mathrm{Be} 1-\mathrm{O} 7-\mathrm{Al}_{3} \mathrm{~A}^{\mathrm{i}}$

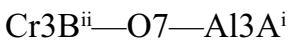

$\mathrm{Al} 3 \mathrm{~A}^{\mathrm{ii}}-\mathrm{O} 7-\mathrm{Al}^{\mathrm{A}} \mathrm{A}^{\mathrm{i}}$
96.34 (3)

121.23 (2)

121.23 (2)

$121.23(2)$

$121.23(2)$

$119.86(4)$

$121.23(2)$

121.23 (2)

121.23 (2)

$121.23(2)$

$119.86(4)$

0.0

97.03 (3)

97.03 (3)

0.0

97.03 (3)

95.4

$95.38(3)$

120.65 (4)

120.46 (2)

120.46 (2)

120.46 (2)

120.65 (4)

120.46 (2)

120.46 (2)

120.46 (2)

0.0

0.0

96.97 (4)

96.97 (4)

97.0

96.97 (4)

0.0

97.0

96.97 (4)

97.0

96.97 (4)

120.16 (3)

120.16 (3)

$120.16(3)$

$120.16(3)$

$120.16(3)$

121.57 (3)

121.57 (3)

0.0

121.57 (3)

95.1

95.10 (4) 


\begin{tabular}{|c|c|}
\hline $\mathrm{A} 14 \mathrm{~A}^{\mathrm{v}}-\mathrm{A} 15-\mathrm{Cr} 4 \mathrm{~B}^{\mathrm{iv}}$ & $59.719(10)$ \\
\hline $\mathrm{O}^{\mathrm{iii}}-\mathrm{A} 15-\mathrm{A} 14 \mathrm{~A}^{\mathrm{iv}}$ & $138.301(15)$ \\
\hline $\mathrm{O}^{\mathrm{ii}}-\mathrm{A} 15-\mathrm{Al}_{4} \mathrm{~A}^{\mathrm{iv}}$ & $94.97(2)$ \\
\hline $\mathrm{O} 5^{\mathrm{i}}-\mathrm{A} 15-\mathrm{A} 14 \mathrm{~A}^{\mathrm{iv}}$ & $138.300(15)$ \\
\hline $\mathrm{O} 4^{\mathrm{iii}}-\mathrm{A} 15-\mathrm{A} 14 \mathrm{~A}^{\mathrm{iv}}$ & $85.22(2)$ \\
\hline $\mathrm{O} 4^{\mathrm{ii}}-\mathrm{A} 15-\mathrm{Al}_{4} \mathrm{~A}^{\mathrm{iv}}$ & $41.825(15)$ \\
\hline $\mathrm{O} 4-\mathrm{i} 15-\mathrm{A} 14 \mathrm{~A}^{\mathrm{iv}}$ & $41.826(15)$ \\
\hline $\mathrm{Cr} \mathrm{B}^{\mathrm{v}}-\mathrm{A} 15-\mathrm{Al} 4 \mathrm{~A}^{\mathrm{iv}}$ & 59.7 \\
\hline $\mathrm{A} 14 \mathrm{~A}^{\mathrm{v}}-\mathrm{A} 15-\mathrm{A} 14 \mathrm{~A}^{\mathrm{iv}}$ & $59.719(10)$ \\
\hline $\mathrm{Cr} 4 \mathrm{~B}^{\mathrm{iv}}-\mathrm{A} 15-\mathrm{A} 14 \mathrm{~A}^{\mathrm{iv}}$ & 0.0 \\
\hline $\mathrm{O}^{\mathrm{iii}}-\mathrm{Al} 5-\mathrm{Cr} 4 \mathrm{~B}^{\mathrm{vi}}$ & $94.97(2)$ \\
\hline $\mathrm{O}^{\mathrm{ii}}-\mathrm{A} 15-\mathrm{Cr} 4 \mathrm{~B}^{\mathrm{vi}}$ & $138.301(15)$ \\
\hline $\mathrm{O} 5^{\mathrm{i}}-\mathrm{A} 15-\mathrm{Cr} 4 \mathrm{~B}^{\mathrm{vi}}$ & $138.301(15)$ \\
\hline $\mathrm{O} 4^{\mathrm{iii}}-\mathrm{A} 15-\mathrm{Cr} 4 \mathrm{~B}^{\mathrm{vi}}$ & $41.826(15)$ \\
\hline $\mathrm{O} 4^{\mathrm{ii}}-\mathrm{A} 15-\mathrm{Cr} 4 \mathrm{~B}^{\mathrm{vi}}$ & $41.826(15)$ \\
\hline $\mathrm{O} 4{ }^{\mathrm{i}}-\mathrm{A} 15-\mathrm{Cr} 4 \mathrm{~B}^{\mathrm{vi}}$ & $85.22(2)$ \\
\hline $\mathrm{Cr} 4 \mathrm{~B}^{\mathrm{v}}-\mathrm{A} 15-\mathrm{Cr} 4 \mathrm{~B}^{\mathrm{vi}}$ & $59.719(10)$ \\
\hline $\mathrm{A} 14 \mathrm{~A}^{\mathrm{v}}-\mathrm{A} 15-\mathrm{Cr} 4 \mathrm{~B}^{\mathrm{vi}}$ & $59.719(10)$ \\
\hline $\mathrm{Cr} 4 \mathrm{~B}^{\mathrm{iv}}-\mathrm{Al} 5-\mathrm{Cr} 4 \mathrm{~B}^{\mathrm{vi}}$ & $59.719(10)$ \\
\hline $\mathrm{Al} 4 \mathrm{~A}^{\mathrm{iv}}-\mathrm{A} 15-\mathrm{Cr} 4 \mathrm{~B}^{\mathrm{vi}}$ & $59.719(10)$ \\
\hline $\mathrm{O} 5^{\mathrm{iii}}-\mathrm{A} 15-\mathrm{A} 14 \mathrm{~A}^{\mathrm{vi}}$ & $94.97(2)$ \\
\hline $\mathrm{O}^{\mathrm{ii}}-\mathrm{A} 15-\mathrm{A} 14 \mathrm{~A}^{\mathrm{vi}}$ & $138.301(15)$ \\
\hline $\mathrm{O}^{\mathrm{i}}-\mathrm{A} 15-\mathrm{A} 14 \mathrm{~A}^{\mathrm{vi}}$ & $138.301(15)$ \\
\hline $\mathrm{O} 4^{\mathrm{iii}}-\mathrm{A} 15-\mathrm{A} 14 \mathrm{~A}^{\mathrm{vi}}$ & $41.826(15)$ \\
\hline $\mathrm{O} 4^{\mathrm{ii}}-\mathrm{A} 15-\mathrm{A} 14 \mathrm{~A}^{\mathrm{vi}}$ & $41.826(15)$ \\
\hline $\mathrm{O} 4 \mathrm{i}-\mathrm{A} 15-\mathrm{A} 14 \mathrm{~A}^{\mathrm{vi}}$ & $85.22(2)$ \\
\hline $\mathrm{Cr} 4 \mathrm{~B}^{\mathrm{v}}-\mathrm{A} 15-\mathrm{A} 4 \mathrm{~A}^{\mathrm{vi}}$ & 59.7 \\
\hline $\mathrm{A} 14 \mathrm{~A}^{\mathrm{v}}-\mathrm{A} 15-\mathrm{A} 14 \mathrm{~A}^{\mathrm{vi}}$ & $59.719(10)$ \\
\hline $\mathrm{Cr} 4 \mathrm{~B}^{\mathrm{iv}}-\mathrm{A} 15-\mathrm{A} 14 \mathrm{~A}^{\mathrm{vi}}$ & 59.7 \\
\hline $\mathrm{A} 14 \mathrm{~A}^{\mathrm{iv}}-\mathrm{A} 15-\mathrm{A} 14 \mathrm{~A}^{\mathrm{vi}}$ & $59.719(10)$ \\
\hline $\mathrm{Cr} 4 \mathrm{~B}^{\mathrm{vi}}-\mathrm{Al} 5-\mathrm{Al}_{4} \mathrm{~A}^{\mathrm{vi}}$ & 0.0 \\
\hline
\end{tabular}

$\mathrm{Be} 1-\mathrm{O} 7-\mathrm{Cr} 3 \mathrm{~B}^{\mathrm{i}}$

$\mathrm{Cr} 3 \mathrm{~B}^{\mathrm{ii}}-\mathrm{O} 7-\mathrm{Cr} \mathrm{B}^{\mathrm{i}}$

$\mathrm{Al} 3 \mathrm{~A}^{\mathrm{ii}}-\mathrm{O} 7-\mathrm{Cr} 3 \mathrm{~B}^{\mathrm{i}}$

$\mathrm{A} 13 \mathrm{~A}^{\mathrm{i}}-\mathrm{O} 7-\mathrm{Cr} 3 \mathrm{~B}^{\mathrm{i}}$

$\mathrm{Be} 1-\mathrm{O} 7-\mathrm{Cr} 3 \mathrm{~B}^{\mathrm{iii}}$

$\mathrm{Cr} 3 \mathrm{~B}^{\mathrm{ii}}-\mathrm{O} 7-\mathrm{Cr} 3 \mathrm{~B}^{\mathrm{iii}}$

$\mathrm{Al} 3 \mathrm{~A}^{\mathrm{ii}}-\mathrm{O} 7-\mathrm{Cr} 3 \mathrm{~B}^{\mathrm{iii}}$

$\mathrm{A} 3 \mathrm{~A}^{\mathrm{i}}-\mathrm{O} 7-\mathrm{Cr} 3 \mathrm{~B}^{\mathrm{iii}}$

$\mathrm{Cr} 3 \mathrm{~B}^{\mathrm{i}}-\mathrm{O} 7-\mathrm{Cr} 3 \mathrm{~B}^{\mathrm{iii}}$

$\mathrm{Be} 1-\mathrm{O} 7-\mathrm{Al} 3 \mathrm{~A}^{\mathrm{iii}}$

$\mathrm{Cr} 3 \mathrm{~B}^{\mathrm{ii}}-\mathrm{O} 7-\mathrm{A} 3 \mathrm{~A}^{\mathrm{iii}}$

$\mathrm{Al} 3 \mathrm{~A}^{\mathrm{ii}}-\mathrm{O} 7-\mathrm{Al} 3 \mathrm{~A}^{\mathrm{iii}}$

$\mathrm{Al} 3 \mathrm{~A}^{\mathrm{i}}-\mathrm{O} 7-\mathrm{A} 13 \mathrm{~A}^{\mathrm{iii}}$

$\mathrm{Cr} 3 \mathrm{~B}^{\mathrm{i}}-\mathrm{O} 7-\mathrm{A} 13 \mathrm{~A}^{\mathrm{iii}}$

$\mathrm{Cr} 3 \mathrm{~B}^{\mathrm{iii}}-\mathrm{O} 7-\mathrm{Al} 3 \mathrm{~A}^{\mathrm{iii}}$

$\mathrm{A} 12^{\mathrm{xix}}-\mathrm{O} 8-\mathrm{Cr}^{3} \mathrm{~B}^{\mathrm{x}}$

$\mathrm{A} 12^{\mathrm{xix}}-\mathrm{O} 8-\mathrm{A} 13 \mathrm{~A}^{\mathrm{x}}$

$\mathrm{Cr} 3 \mathrm{~B}^{\mathrm{x}}-\mathrm{O} 8-\mathrm{A} 13 \mathrm{~A}^{\mathrm{x}}$

$\mathrm{Al} 2^{\mathrm{xix}}-\mathrm{O} 8-\mathrm{A} 13 \mathrm{~A}^{\mathrm{xiii}}$

$\mathrm{Cr} 3 \mathrm{~B}^{\mathrm{x}}-\mathrm{O} 8-\mathrm{A} 3 \mathrm{~A}^{\mathrm{xiii}}$

$\mathrm{A} 13 \mathrm{~A}^{\mathrm{x}}-\mathrm{O} 8-\mathrm{A} 13 \mathrm{~A}^{\mathrm{xiii}}$

$\mathrm{A} 12^{\mathrm{xix}}-\mathrm{O} 8-\mathrm{Cr} 3 \mathrm{~B}^{\mathrm{xiii}}$

$\mathrm{Cr} 3 \mathrm{~B}^{\mathrm{x}}-\mathrm{O} 8-\mathrm{Cr} 3 \mathrm{~B}^{\mathrm{xiii}}$

$\mathrm{A} 13 \mathrm{~A}^{\mathrm{x}}-\mathrm{O} 8-\mathrm{Cr} 3 \mathrm{~B}^{\mathrm{xiii}}$

$\mathrm{Al} 3 \mathrm{~A}^{\mathrm{xii}}-\mathrm{O} 8-\mathrm{Cr} 3 \mathrm{~B}^{\mathrm{xiii}}$

$\mathrm{A} 12^{\mathrm{xix}}-\mathrm{O} 8-\mathrm{Mg} 3^{\text {xiii }}$

$\mathrm{Cr} 3 \mathrm{~B}^{\mathrm{x}}-\mathrm{O} 8-\mathrm{Mg}^{\mathrm{xiii}}$

$\mathrm{Al} 3 \mathrm{~A}^{\mathrm{x}}-\mathrm{O} 8-\mathrm{Mg}^{\mathrm{xiii}}$

$\mathrm{A} 13 \mathrm{~A}^{\mathrm{xiii}}-\mathrm{O} 8-\mathrm{Mg} 3^{\text {xiii }}$

$\mathrm{Cr} 3 \mathrm{~B}^{\text {xiii }}-\mathrm{O} 8-\mathrm{Mg}^{\text {xiii }}$
121.57 (3)

$95.10(4)$

$95.10(4)$

$0.000(18)$

121.57 (3)

$95.10(4)$

$95.10(4)$

$95.10(4)$

$95.10(4)$

121.57 (3)

95.1

$95.10(4)$

$95.10(4)$

95.1

0.0

$123.84(2)$

$123.84(2)$

0.0

123.84 (2)

95.5

95.47 (4)

123.84 (2)

95.47 (4)

95.47 (4)

0.000 (17)

116.60 (4)

95.04 (3)

95.04 (3)

95.04 (3)

95.04 (3)

Symmetry codes: (i) $x+1, y+1, z$; (ii) $-y, x-y+1, z$; (iii) $-x+y,-x, z$; (iv) $-y, x-y, z$; (v) $x, y+1, z$; (vi) $-x+y+1,-x+1, z$; (vii) $-x,-y,-z$; (viii) $x+1, y, z$; (ix) $x-y+1, x+1,-z$; (x) $-x+y,-x-1, z$; (xi) $-x+1,-y,-z$; (xii) $-x+1,-y+1,-z$; (xiii) $x, y-1, z$; (xiv) $-y, x-y-1, z$; (xv) $-y+1, x-y, z$; (xvi) $-x+y+1,-x, z$; (xvii) $-x+y-1,-x-1, z$; (xviii) $-y-1, x-y-1, z$; (xix) $x-1, y-1, z$; (xx) $-y-1, x-y, z$; (xxi) $-x+1,-y,-z+1$; (xxii) $x-y+1, x+1,-z+1$; (xxiii) $-x,-y,-z+1$; (xxiv) $-y+1, x-y+1, z ;(\mathrm{xxv}) x-1, y, z$. 